\title{
DYNAMIC PRICING OF GENERAL INSURANCE IN A COMPETITIVE MARKET
}

\author{
BY \\ Paul EMms
}

\begin{abstract}
A model for general insurance pricing is developed which represents a stochastic generalisation of the discrete model proposed by Taylor (1986). This model determines the insurance premium based both on the breakeven premium and the competing premiums offered by the rest of the insurance market. The optimal premium is determined using stochastic optimal control theory for two objective functions in order to examine how the optimal premium strategy changes with the insurer's objective. Each of these problems can be formulated in terms of a multi-dimensional Bellman equation.

In the first problem the optimal insurance premium is calculated when the insurer maximises its expected terminal wealth. In the second, the premium is found if the insurer maximises the expected total discounted utility of wealth where the utility function is nonlinear in the wealth. The solution to both these problems is built-up from simpler optimisation problems. For the terminal wealth problem with constant loss-ratio the optimal premium strategy can be found analytically. For the total wealth problem the optimal relative premium is found to increase with the insurer's risk aversion which leads to reduced market exposure and lower overall wealth generation.
\end{abstract}

\section{INTRODUCTION}

Dynamic programming has found widespread application in both finance and insurance originating with the optimal asset allocation problem (Samuelson 1969, Merton 1971; 1990). Merton found the optimal allocation of resources in a portfolio consisting of a risky asset and a risk-free asset in order to maximise some measure of wealth. Part of the attraction of the theory is that the optimal strategy can be determined in closed-form under certain assumptions concerning the form of the process describing the risky asset and the objective of the portfolio manager. The strategy is dynamic since it uses information as it arises: the optimal strategy can be determined as long as we specify the distributions of the underlying processes and the current values of the state variables. There are many generalisations of the problem in the literature including, for example, an $N$ dimensional state space, non-Markov underlying processes, 
and random time horizons. Reviews of the literature are contained in Karatzas \& Shreve (1998) and Campbell \& Viceira (2001).

In actuarial science there are a number of established problems which have been tackled using dynamic programming. The optimal asset allocation problem for an insurer was investigated by Browne (1995). The problem is more complex for an insurance company because it invests in risky assets in order to maintain a reserve to pay out incoming claims. There are then at least two, possibly correlated, random processes required for the model: the invested asset and the claims process. Browne used a normal random process to model the cash flow and found it is optimal to invest a fixed amount of the reserve if the objective is to maximise the expected utility of wealth for an exponential utility function. He also found that this strategy corresponds to minimising the probability of ruin when the risk-free interest rate is zero. Hipp \& Plum (2000) considered the problem with a compound Poisson process for the claims process: the optimal strategy now depends on the current value of the insurer's reserve. Other insurance problems which use dynamic programming include optimal proportional reinsurance (Højgaard \& Taksar 1997), optimal new business generation (Hipp \& Taksar 2000) and optimal dividend payout for an insurer (Asmussen \& Taksar 1997). A comprehensive review of optimal control theory applied to insurance can be found in Hipp (2004).

In this paper we determine the optimal premium strategy for an insurer which maximises a particular objective over a fixed planning horizon. Actuaries traditionally price insurance through a premium principle, which relates the charge for cover to the moments of the claims distribution. In this work we determine the premium by using a competitive demand model (following Taylor 1986) as well as the expected claim size. Consequently, we suppose that the premium is determined in part by the price relative to the rest of the insurance market. It is not enough that an insurer set a price to cover claims if the rest of the market undercuts that price since then the insurer will gain insufficient income to remain viable.

The demand law specifies how the insurer's income and exposure change with the relative to market premium: a low relative premium generates exposure but leads to reduced premium income. The approach builds on the work in Emms, Haberman \& Savoulli (2007) and Emms \& Haberman (2005), but develops a more sophisticated and realistic model, so that the dependence of the optimal strategy on the current state is stochastic. Emms, Haberman \& Savoulli (2007) developed a continuous time version of Taylor's model (Taylor 1986) because continuous time control systems are often easier to analyse than discrete problems. However, the continuous time version of Taylor's model leads to a bang-bang optimal premium strategy which is not realistic. Consequently, Emms \& Haberman (2005) developed an accrued premium pricing model by supposing that the continuously varying premium is paid at a rate fixed at the start of the policy. Here, we also suppose that the premium is a continuous process; however, policyholders are assumed to pay a fixed amount at the start of the policy in return for a constant period of cover. A renewal of insurance is treated as if the policyholder were buying a new period of cover. This assumption reduces the state space of the optimisation problem and 
so makes the analysis easier. Emms \& Haberman (2005) determined the optimal premium strategy which maximised the expected terminal wealth of the insurer. We examine a terminal wealth and a total wealth objective and assess the qualitative impact of this change on the optimal premium strategy.

In Section 2 we propose a two factor model of the general insurance market: one factor models the randomness of the claim size and intensity, whilst the other models the market average premium. This model is used to determine the premium which maximises a number of possible objective functions of the insurer. Section 3 describes the derivation of the Bellman equation which gives the optimal dynamic premium strategy to maximise the expected terminal wealth of the insurer. The problem reduces to the solution of a reaction-diffusion equation, which is straightforward to solve numerically. Next in Section 4 we consider the objective of maximising the expected total discounted utility of wealth with a utility function linear in the wealth. This is probably a more realistic objective for an insurer given the regulatory constraints imposed over the course of the planning horizon. The resulting reaction-diffusion equation is slightly more complex but it is again straightforward to solve numerically. We consider a nonlinear utility function by constructing a perturbation expansion in the risk aversion of the insurer. This leads to a pair of coupled reaction-diffusion equations and a numerical solution gives the optimal premium strategy. Finally in Section 5 we compare the strategies and suggest further work.

\section{MARKOV MODEL}

Emms, Haberman \& Savouilli (2007) extended Taylor's model to the case that the market average premium is stochastic and the breakeven premium is constant. Here we generalise further by adopting stochastic processes with given distributions for the market average premium $\overline{\mathbf{p}}_{t}$ (per unit exposure) and the loss-ratio $\gamma_{t}$. Since the market average premium is positive we suppose that it is lognormally distributed:

$$
d \overline{\mathbf{p}}_{t}=\overline{\mathbf{p}}_{t}\left(\mu d t+\sigma_{1} d \mathbf{W}_{1 t}\right),
$$

where the drift $\mu$ and the coefficient of volatility $\sigma_{1}$ are constants and $\mathbf{W}_{1 t}$ is a Brownian motion. Note that all premiums are specified as per unit of exposure so that each policyholder pays the same premium irrespective of the exposure that is being insured. Hereafter we shall omit this qualification.

We introduce the relative loss ratio which we define as the ratio between the breakeven premium $\pi_{t}$ of the insurer and the market average premium ${ }^{1}$ :

$$
\gamma_{t}=\frac{\pi_{t}}{\overline{\mathbf{p}}_{t}}
$$

\footnotetext{
1 In the actuarial literature the loss ratio is usually defined as the claims divided by the premiums
} received by a company over a year (Daykin et al. 1994). 
The breakeven premium is the random amount a policy of length $\tau$ costs the insurance company. This is the actuarial premium without any profit margin and can be deduced from the insurer's previous claims data and a loading factor to account for expenses and interest rates (Daykin \& Hey 1990). Rather than complicating the model with a precise formulation for this factor we suppose that the breakeven premium is a stochastic process (Pentikäinen 1986). The advantage of this formulation is that there are no outstanding liabilities at the end of the planning horizon $T$ because as soon as policies of total exposure $\delta \mathbf{q}_{t}$ are bought, the insurer sets aside $\pi_{t} \delta \mathbf{q}_{t}$ to cover the resulting claims. Appendix A describes the modifications to the theory if we adopt a stochastic process for the mean claim size rate.

We expect a direct correlation between the premium that the market charges and the breakeven premium of the insurer. If we suppose that claims are identically distributed for the market and the individual insurer then the discrepancy between these quantities represents the different loadings that insurance companies apply to their policies. Consequently we suppose that the log of the loss-ratio is a Vasicek process ( $\gamma_{t}$ must be positive) and that the mean of $\log \gamma_{t}$ reverts to zero:

$$
d \log \gamma_{t}=-\rho \log \gamma_{t} d t+\sigma_{2} d \mathbf{W}_{2 t},
$$

where $\rho$ is the rate of reversion, $\sigma_{2}$ is the coefficient of volatility and $\mathbf{W}_{2 t}$ is a Brownian motion independent of $\mathbf{W}_{1 t}$. Ito's Lemma gives the SDE for $\gamma_{t}$ as

$$
d \gamma_{t}=\gamma_{t}\left[\frac{1}{2} \sigma_{2}^{2}-\rho \log \gamma_{t}\right] d t+\sigma_{2} \gamma_{t} d \mathbf{W}_{2 t} .
$$

This definition forces the market average premium towards the breakeven premium: $\mathbb{E}\left[\gamma_{t}\right]=e^{\sigma_{2}^{2} / 4 \rho}$ as $t \rightarrow \infty$. Market data often show the market average premium drifting above and below the breakeven premium so this would seem to be a reasonable model (Daykin et al. 1994).

Suppose a policyholder pays a premium $p(t)$ to the insurer at the start of the policy for a fixed term of insurance cover $\tau$. We suppose that at the end of the cover the policyholder can renew by paying $p(t+\tau)$, that is the same premium as that charged to new business. We suppose the insurer writes a number of these policies in a time interval $\delta t$ generating total exposure $\delta \mathbf{q}_{t}$ and receives the premium income $\mathbf{p}_{t} \delta \mathbf{q}_{t}$ in return. Thus $\mathbf{p}_{t}$ is the premium charged to new policyholders and existing policyholders who want to renew their policies.

The model is completed by evolution equations for the insurer's exposure $\mathbf{q}_{t}$ and wealth $\mathbf{w}_{t}$. We suppose that the rate of generation of new and renewed exposure $\mathbf{b}_{t}$ is parameterised as

$$
\mathbf{b}_{t}=\mathbf{q}_{t} G\left(\mathbf{k}_{t}\right),
$$

where $G$ is a demand function which represents the fractional increase in exposure per unit time arising from the relative premium charged, $\mathbf{k}_{t}$, defined by

$$
\mathbf{k}_{t}=\mathbf{p}_{t} / \overline{\mathbf{p}}_{t} .
$$


Consequently the wealth generated by premium income over time $\delta t$ due to a fractional increase in exposure is $\mathbf{p}_{t} \delta \mathbf{q}_{t}=\mathbf{p}_{t} \mathbf{b}_{t} \delta t$.

This notation leads to the following SDEs:

$$
\begin{aligned}
& d \mathbf{q}_{t}=\left(\mathbf{b}_{t}-\kappa \mathbf{q}_{t}\right) d t, \\
& d \mathbf{w}_{t}=-\alpha \mathbf{w}_{t} d t+\left(\mathbf{p}_{t}-\boldsymbol{\pi}_{t}\right) \mathbf{b}_{t} d t,
\end{aligned}
$$

where $\kappa>0$ is a constant representing the fractional loss of exposure due to non-renewed business and $\alpha>0$ represents the loss of wealth due to returns paid to shareholders. In reality, this loss of exposure is related to the number of policies initiated at times $t-\tau, t-2 \tau, \ldots$ since it is only these policyholders who make a renewal decision at time $t$. Models incorporating this feature lead to either high dimensional state spaces or non-Markov processes both of which considerably complicate the optimisation theory and are not considered here (see Kolmanovskiı̆ \& Shaĭkhet 1996). In practice $\kappa$ must be determined by data on policy renewals: for simplicity we set $\kappa=\tau^{-1}$.

The main difference between this model and that presented in Emms \& Haberman (2005) is the interpretation of the "new business" parameterisation. This means that the term $\mathbf{p}_{t} \mathbf{b}_{t} d t$ appears in the wealth equation (6) reflecting the idea that it is only new business and renewed business who pay a premium not the entire customer base. The simplification arises because we set a premium at the start of the policy rather than a premium rate which varies over the course of the policy. The claims arising from insurance taken out at time $t$ are accounted for by the term $\pi_{t} \mathbf{b}_{t}$ in (6): claims on existing exposure were accounted for when these policies were initiated.

\section{Terminal Wealth}

The premium strategy is determined by maximising the objective function of the insurer. Due to the parameterisation of the demand law it is simpler if we use the relative premium $\mathbf{k}_{t}$ as the control in the dynamic optimisation problem. Thus, first we aim to find the optimal relative premium $k^{*}$ which maximises the insurer's wealth at the end of the planning horizon $T$. We define the value function

$$
V(x, t)=\sup _{k} \mathbb{E}\left[\mathbf{w}_{\mathcal{T}} \mid \mathbf{X}_{t}=x\right]
$$

where the state of the system is $\mathbf{X}_{t}=\left(\overline{\boldsymbol{p}}_{t}, \mathbf{q}_{t}, \mathbf{w}_{t}, \boldsymbol{\gamma}_{t}\right)$, the current state is $x=(\bar{p}, q$, $w, \gamma)$, and for the moment $\mathcal{T}=T$. If the value function is sufficiently smooth then it satisfies the Bellman equation

$$
\begin{array}{r}
V_{t}+\mu \bar{p} V_{\bar{p}}+\frac{1}{2} \sigma_{1}^{2} \bar{p}^{2} V_{\bar{p} \bar{p}}+\gamma\left(\frac{1}{2} \sigma_{2}^{2}-\rho \log \gamma\right) V_{\gamma}+\frac{1}{2} \sigma_{2}^{2} \gamma^{2} V_{\gamma \gamma}-\kappa q V_{q}-\alpha w V_{w}+ \\
q \sup _{k}\left\{G(k)\left(V_{q}+\bar{p}(k-\gamma) V_{w}\right)\right\}=0,
\end{array}
$$


with boundary condition

$$
V(x, T)=w .
$$

The first-order condition for a maximum of $k$ is

$$
k+\frac{G(k)}{G^{\prime}(k)}=\gamma-\frac{V_{q}}{\bar{p} V_{w}},
$$

where' denotes differentiation wrt. $k$. The control determined by this first order condition is called interior and is denoted with a superscript $i$. The control which satisfies the maximisation operation of the Bellman equation is denoted by $k^{*}$, and may not equal the interior control $k^{i}$. For simplicity, we adopt a linear demand function (Lilien \& Kotler 1983):

$$
G(k)= \begin{cases}a(b-k) & \text { if } k \leq b, \\ 0 & \text { if } k>b,\end{cases}
$$

where $a>0$ has dimension of per unit time and $b \geq 1$ is dimensionless. Notice that there is no lower bound on the control. The interior relative premium is now given by

$$
k^{i}=\frac{1}{2}\left(b+\gamma-\frac{V_{q}}{\bar{p} V_{w}}\right)
$$

providing that $\gamma \leq b+V_{q} / \bar{p} V_{w}$.

Since $V_{q}=0$ and $V_{w}=1$ at $t=T$, the terminal interior relative premium is

$$
k_{T}^{i}=\frac{1}{2}(b+\gamma) \text { if } \gamma \leq b .
$$

The terminal interior premium is at least the breakeven premium $p_{T}^{i} \geq \pi$ since $k_{T}^{i} \geq \gamma$. If $\gamma>b$, that is $\pi>b \bar{p}$, then the interior premium is undefined. In this case, the insurer can set any relative premium $k>b$ since no-one will buy insurance at this price according to the demand law (9). In such circumstances we set $k^{*}=b$ for continuity of the control.

\subsection{Constant loss ratio}

We need the results from this section to determine the appropriate boundary condition for (8) when $\gamma$ is stochastic. Suppose the loss ratio $\gamma$ is constant corresponding to setting $\rho=\sigma_{2}=0$. Let us look for a value function of the form

$$
V(x, t)=e^{\alpha(t-T)}\left(w+q \bar{p} f_{0}(t)\right) .
$$


Substituting this expression into the Bellman equation (8) yields

$$
\frac{d f_{0}}{d t}+(\mu+\alpha-\kappa) f_{0}+\sup _{k}\left\{G(k)\left(f_{0}+k-\gamma\right)\right\}=0,
$$

with boundary condition $f_{0}(T)=0$. The interior control is given by

$$
k_{0}^{i}(t)=\frac{1}{2}\left(b+\gamma-f_{0}(t)\right) .
$$

At termination $k_{0}^{i}(T)=\frac{1}{2}(b+\gamma)$. If $\gamma>b$ then $k^{*}(T)=b$ and $G^{*}=0$. Consequently, the Bellman equation integrates immediately to give $f_{0} \equiv 0$ and so $k^{*}(t) \equiv b$ for $t \in[0, T]$. This means the optimal strategy is to set a price so that no insurance is ever sold. It is easy to see why this is optimal: the insurer only generates wealth from insurance if $k>\gamma>b$ from the wealth equation. But at this relative premium no exposure is generated, so the insurer can never make a profit from selling insurance.

If $\gamma \leq b$ then at termination $k^{*}(T)=k_{0}^{i}(T)$. Substituting the interior control into the Bellman equation (13) yields

$$
\frac{d f_{0}}{d t}+\frac{1}{4} a f_{0}^{2}+A f_{0}+B=0
$$

where

$$
\begin{aligned}
A(\gamma) & =\frac{1}{2} a(b-\gamma)+\phi, \\
B(\gamma) & =\frac{1}{4} a(b-\gamma)^{2}, \\
\phi & =\mu+\alpha-\kappa .
\end{aligned}
$$

Note that $\gamma$ is constant and so therefore $A(\gamma)$ and $B(\gamma)$ are also constants. On integrating (15) and applying the boundary condition we obtain

$$
f_{0}(t ; \gamma)= \begin{cases}\frac{2}{a}\left(D_{+} \tan \left(\frac{T-t}{2} D_{+}+\tan ^{-1}\left(\frac{A}{D_{+}}\right)\right)-A\right) & \text { if } a B>A^{2}, \\ \frac{2}{a}\left(\frac{E(t)\left(D_{-}+A\right)+D_{-}-A}{1-E(t)}\right) & \text { if } a B<A^{2}, \\ \frac{2 A^{2}(T-t)}{a(2-A(T-t))} & \text { if } a B=A^{2},\end{cases}
$$

where

$$
E(t)=\left(\frac{A-D_{-}}{A+D_{-}}\right) \exp \left((T-t) D_{-}\right)
$$


and

$$
D_{ \pm}^{2}= \pm a B \mp A^{2}=\mp \phi(a(b-\gamma)+\phi) .
$$

At termination $f_{0}(T)=0$ and from (15), $d f_{0} / d t \leq 0$ so that as we integrate from $t=T$ towards $t=0, f_{0}$ increases and it is positive. Further, if $f_{0}$ is continuous then it must remain positive because if at any point $f_{0}=0$ then $d f_{0} / d t \leq 0$ and $f_{0}$ increases. Consequently, $k^{*}(t)=k_{0}^{i}(t) \leq k_{0}^{i}(T)=\frac{1}{2}(b+\gamma)$ providing that $f_{0}(t)$ remains continuous in $[0, T]$.

The value function $V$, given by (12), is twice continuously differentiable in the state variables and once continuously differentiable in time (under parametric restrictions). Moreover $k^{*}(t)$, by definition, does satisfy the maximisation operator of the Bellman equation, and it is admissible because the state equations are linear in the state variables. Consequently, $k^{*}(t)$ is the optimal relative premium strategy by Verification Theorem 4.1 of Fleming \& Rishel (1975) as long as $f_{0} \in C^{1}[0, T]$. This premium strategy corresponds to that found in Emms \& Haberman (2005), although here we are able to give an analytical expression for the strategy because of the simplification of the model. It is only the first case in (17) that leads to discontinuities, which corresponds to the blow-up in the Ricatti equation of the accrued premium model. Specifically, there is blowup at $t_{b}$ if

$$
t_{b}=T-\frac{2}{D_{+}}\left(\frac{\pi}{2}-\tan ^{-1}\left(\frac{A}{D_{+}}\right)\right) \in[0, T] .
$$

If this is the case then $f_{0}\left(t_{b}\right)=\infty$ and the optimal control is not smooth.

\subsection{Stochastic loss ratio}

When the loss ratio is stochastic we are unable to solve the Bellman equation analytically. Motivated by the previous section we introduce the stopping time

$$
\tau=\inf \left\{t>0: \gamma_{t}=b\right\}
$$

and set

$$
\mathcal{T}=\tau \wedge T
$$

Consequently, we bound the sample paths so that the loss ratio does not become too large. We have chosen to bound the loss ratio by $b$ because if $\gamma_{t}>b$ then there is no relative premium which generates immediate wealth for the insurer. Essentially, we restrict sample paths to those for which the insurer actively sells insurance: the insurer cannot leave the market and then return should conditions become favourable. The Bellman equation for this value function is identical to (8) providing that it is sufficiently smooth (see Fleming \& Rishel (1975) p. 154). 
However, now the state space is restricted to $0 \leq \gamma \leq b$ and on the boundary $\gamma=b$ we have $V=w$.

If the optimal control is given by the first order condition then substituting (10) into the Bellman equation (8) we find

$$
\begin{aligned}
V_{t}+\mu \bar{p} V_{\bar{p}}+ & \frac{1}{2} \sigma_{1}^{2} \bar{p}^{2} V_{\bar{p} \bar{p}}+\gamma\left(\frac{1}{2} \sigma_{2}^{2}-\rho \log \gamma\right) V_{\gamma}+\frac{1}{2} \sigma_{2}^{2} \gamma^{2} V_{\gamma \gamma}+ \\
& q\left(\frac{1}{2} a(b-\gamma)-\kappa\right) V_{q}+\left(-\alpha w+\frac{1}{4} a \bar{p} q(b-\gamma)^{2}\right) V_{w}+\frac{a q V_{q}^{2}}{4 \bar{p} V_{w}}=0,
\end{aligned}
$$

where we have used

$$
G^{i}=\frac{a}{2}\left(b-\gamma+\frac{V_{q}}{\bar{p} V_{w}}\right)
$$

The Bellman equation (21) determines the evolution of the value function when the optimal control is interior, that is, it is given by (10).

Assuming that the solution to the interior equation is sufficiently smooth, a Taylor series expansion yields at $t=T-\delta t$

$$
V_{T-\delta t}=w+\left(-\alpha w+\frac{1}{4} a \bar{p} q(b-\gamma)^{2}\right) \delta t
$$

so that $V_{q}=\frac{1}{4} a \bar{p}(b-\gamma)^{2} \delta t$ and $V_{w}=1-\alpha \delta t$. Consequently, the interior relative premium at $t=T-\delta t$ is

$$
k_{T-\delta t}^{i}=\frac{1}{2}\left(b+\gamma-\frac{1}{4} a(b-\gamma)^{2} \delta t\right)
$$

neglecting terms of $O\left(\delta t^{2}\right)$. This gives the premium strategy close to the end of the planning horizon. It gives us confidence that the premium is well-defined locally using an explicit finite difference scheme and it is not determined by external constraints.

The local expansion (22) suggests that the value function takes the form

$$
V(x, t)=e^{\alpha(t-T)}(w+q \bar{p} f(\gamma, t)) .
$$

If this is the case, the optimal strategy is independent of the coefficient of volatility for the market average premium $\sigma_{1}$. Substituting this expression into the Bellman equation (8) yields a nonlinear PDE for $f$ :

$$
f_{t}+\gamma\left(\frac{1}{2} \sigma_{2}^{2}-\rho \log \gamma\right) f_{\gamma}+\frac{1}{2} \sigma_{2}^{2} \gamma^{2} f_{\gamma \gamma}+\phi f+\sup _{k} M(k, \gamma, t)=0,
$$

where the function $M(k, \gamma, t)$ is defined by

$$
M(k, \gamma, t)= \begin{cases}a(b-k)(f(\gamma, t)+k-\gamma) & \text { if } k \leq b \\ 0 & \text { if } k>b\end{cases}
$$




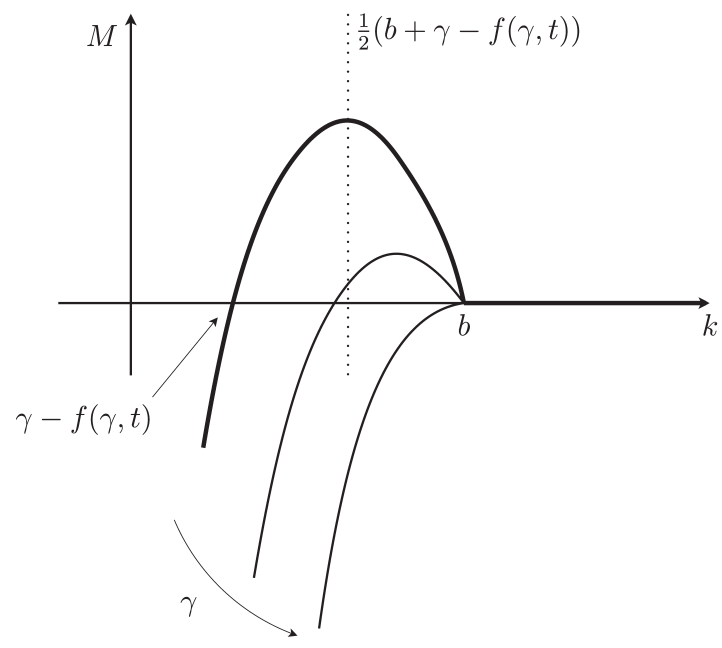

Figure 1: Behaviour of the function $M$ given in (26) as a function of the relative premium $k$ and the loss-ratio $\gamma$ at a fixed time $t$. For $k>b$ the value of $M$ is zero corresponding to a withdrawal from the insurance market.

We require three boundary conditions in order to solve this problem numerically. The first condition arises from the definition of the value function (7): $V=w$ at $t=T$ giving $f(\gamma, T)=0$. If $\gamma=0$ then from the SDE (3) the loss ratio $\gamma_{s} \equiv 0$ for $s \in[t, T]$. Thus the loss ratio is constant and the optimal premium strategy is that derived in Section 3.1. Consequently the appropriate boundary condition on $\gamma=0$ is $f(0, t)=f_{0}(t ; 0)$ where $f_{0}$ is given by (17). The third condition is determined from the stopping time of sample paths: $V=w$ at $t=\tau$ giving $f(b, t)=0$. Figure 1 shows how $M$ varies as we vary the relative premium $k$ for a number of values of the loss-ratio $\gamma$ and at a fixed time $t$. The function $M$ is quadratic in $k$ and has an interior maximum as long as $\gamma \leq b+f(\gamma, t)$. If $f(\gamma$, $t)$ is non-negative then the interior control yields the supremum of (25) in the domain $[0, b] \times[0, T]$.

If $f$ is non-negative the Bellman equation (25) becomes

$$
f_{t}+\gamma\left(\frac{1}{2} \sigma_{2}^{2}-\rho \log \gamma\right) f_{\gamma}+\frac{1}{2} \sigma_{2}^{2} \gamma^{2} f_{\gamma \gamma}+\frac{1}{4} a f^{2}+A(\gamma) f+B(\gamma)=0,
$$

where $A(\gamma)$ and $B(\gamma)$ are given by (16). The boundary conditions for (27) are

$$
f(\gamma, T)=0, \quad f(0, t)=f_{0}(t ; 0), f(b, t)=0 .
$$

Given the solution to equation (27) and its associated boundary conditions (28) the interior relative premium is given by

$$
k^{i}=\frac{1}{2}(b+\gamma-f(\gamma, t)) .
$$


The complexity of the boundary conditions and the nonlinearity of (27) suggests that the problem must be solved numerically. Indeed, the equation is similar to the Fisher equation in Mathematical Biology (Britton 1986, Murray 2002), which is the canonical reaction-diffusion equation. In contrast, (27) has an additional advective term $f_{\gamma}$, the diffusion coefficient is not constant, and the equation is inhomogeneous. One usually looks for travelling wave solutions of the Fisher equation, which reduces the problem to an ODE, but here the spatial domain is restricted so this is not appropriate.

Equation (27) is a semi-linear PDE and it is in the form of an initial-boundary value problem. Friedman (1964) gives existence and uniqueness results for this class of problem. Specifically, if there is a solution of the equation then it is unique by Theorem 8 on p. 41, which follows from the weak Maximum Principle for Parabolic PDEs. Friedman (1964) proves a global existence theorem for semilinear PDEs providing a linear growth condition is satisfied. The quadratic term $f^{2}$ in (27) prevents the application of this result. A general global existence result is clearly not available since we know the boundary condition can blowup at time $t_{b}$ dependent on the model parameters. Consequently, we must be content with the local existence result of Theorem 10 on p. 206, which means the equation has a solution providing the time horizon $T$ is sufficiently small.

If the numerical solution is robust with respect to grid spacing then it seems reasonable to assume that the numerical solution does converge to the exact solution of (27). If $f$ is sufficiently smooth, non-negative and bounded on $[0, b] \times[0, T]$ then the verification theorem in Fleming \& Rishel (1975) can be applied so that $k^{i}$ is the optimal control. The qualitative behaviour of the optimal control for the general problem is determined in large part by the variation of $f_{0}$ with the model parameters since this is the only forcing function in the problem. We know that if $t_{b} \in[0, T]$ then $f_{0}$ is unbounded, and so therefore $f$ is unbounded. Consequently, there are parameter sets for which (29) does not yield the optimal control. Next we shall describe the form of $f_{0}$ in terms of a reduced set of non-dimensional parameters.

\subsection{Numerical Solution}

We solve the nondimensionalised problem numerically. There are many possible time scales based on the drift and volatilities of the stochastic processes: we choose the planning horizon $T$ and introduce the non-dimensional time to termination, $s$, given by

$$
t=T(1-s)
$$

This scale leads to the following nondimensional parameter set:

$$
\begin{array}{lll}
\hat{\rho}=\rho T, & \hat{\sigma}_{2}^{2}=\sigma_{2}^{2} T, & \hat{\phi}=\phi T, \\
\hat{a}=a T, & \hat{A}=A T, & \hat{B}=B T,
\end{array}
$$


which has the advantage of reducing the number of free parameters by one. Henceforth we shall drop the hats on the nondimensional parameters for the numerical work in order to simplify the notation. Numerical results are presented solely in terms of nondimensional parameters and variables.

The free parameters are now $\rho, \sigma_{2}, a, b, \phi$ and in terms of these parameters (27) becomes

$$
f_{s}=\gamma\left(\frac{1}{2} \sigma_{2}^{2}-\rho \log \gamma\right) f_{\gamma}+\frac{1}{2} \sigma_{2}^{2} \gamma^{2} f_{\gamma \gamma}+\frac{1}{4} a f^{2}+A(\gamma) f+B(\gamma),
$$

with boundary conditions

$$
f(0, s)-f_{0}(s ; 0)=f(\infty, s)=f(\gamma, 0)=0 .
$$

In the first case of (17), the governing inequality is $\phi(a b+\phi)<0$ and since $a b$ is positive then this condition becomes $a b>-\phi>0$. Therefore (17) becomes

$$
f_{0}(s ; 0)= \begin{cases}\frac{2}{a}\left(D_{0+} \tan \left(\frac{1}{2} s D_{0+}+\tan ^{-1}\left(\frac{A_{0}}{D_{0+}}\right)\right)-A_{0}\right) & \text { if } a b>-\phi>0, \\ \frac{2}{a}\left(\frac{E(s)\left(D_{0-}+A_{0}\right)+D_{0-}-A_{0}}{1-E_{0}(s)}\right) & \text { if } \phi(a b+\phi)>0, \\ \frac{2 A_{0}^{2} s}{a\left(2-A_{0} s\right)} & \text { if } \phi=0 \text { or } a b=-\phi .\end{cases}
$$

The remaining notation is from (18) and (19) and we have used the subscript zero to denote that the quantity must be evaluated at $\gamma=0$. We find that for the numerical values quoted in Table $1, a b>-\phi>0$ so that the expression for $f_{0}$ is given by the first of these cases.

TABLE 1

SAMPLE DATA SET

\begin{tabular}{ll}
\hline Time horizon $T$ & $2 \mathrm{yr}$ \\
Depreciation of wealth $\alpha$ & 0.05 p.a. \\
Discount factor $\beta$ & 0.05 p.a. \\
Demand parameterisation $a$ & 1.5 p.a. \\
Demand parameterisation $b$ & 1 \\
Length of policy $\tau=\kappa^{-1}$ & $1 \mathrm{yr}$ \\
Market average premium growth $\mu$ & 0.1 p.a. \\
Market average premium volatility $\sigma_{1}$ & 0.1 p.a. \\
Loss ratio mean reversion $\rho$ & 0.1 p.a. \\
Loss ratio volatility $\sigma_{2}$ & 0.1 p.a. \\
\hline
\end{tabular}




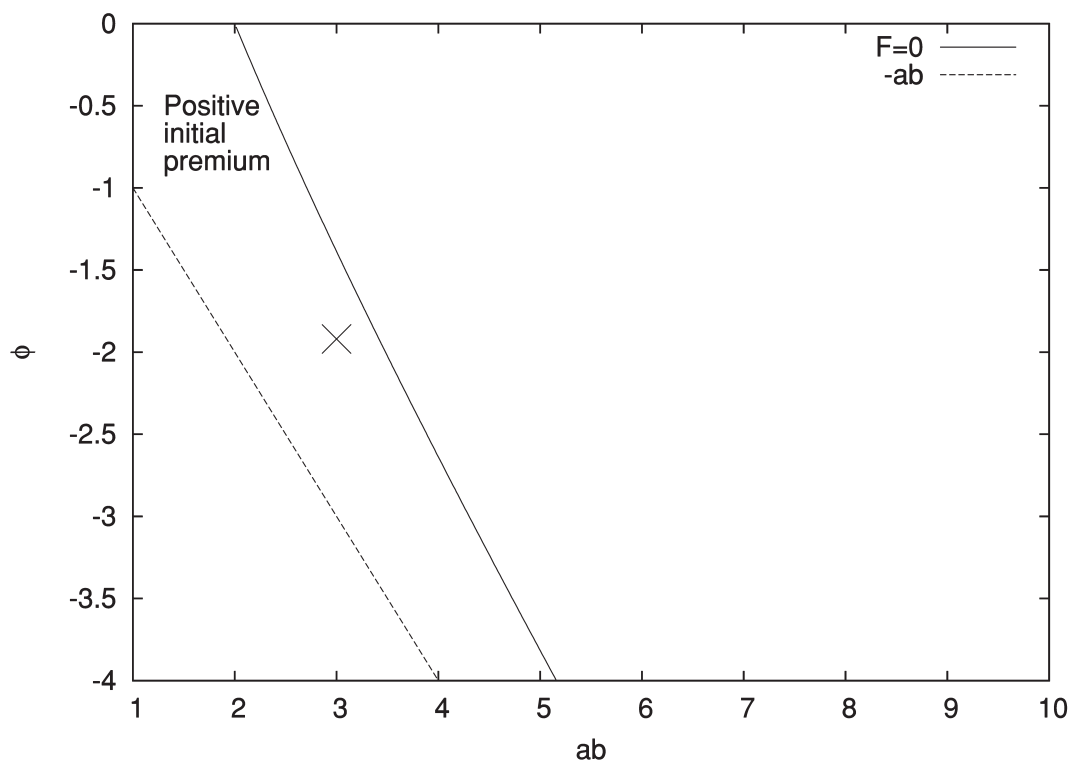

FIgURE 2: Parameteric conditions for a positive initial premium if $f_{0}$ is given by the first expression in (17), which requires $\phi>-a b$. In the figure we show the contour $F=0$ where $F$ is given by (34) as a function of $\phi$ and $a b$. To the left of the contour the parameters yield a positive value of $f_{0}(0 ; 0)$. The cross represents the parameters given in Table 1.

It is clear from (29) that we must have $f_{0}(s ; 0)<b$ for a positive interior premium. If we suppose that $f_{0}$ is given by the first alternative in (33) then this condition is at $s=1$ (corresponding to $t=0$ )

$$
F(a b, \phi):=\tan ^{-1}\left(\frac{\frac{1}{2} a b+\phi}{D_{0+}}\right)-\tan ^{-1}\left(\frac{a b+\phi}{D_{0+}}\right)-\frac{1}{2} D_{0+}>0,
$$

Figure 2 shows a plot of the contour $F=0$ for a range of values of $a b$ and $\phi$. Only the parameter set containing the cross in the figure lead to a positive optimal premium at $t=0$.

Table 1 gives a sample data set to solve the problem numerically. The planning horizon $T$ is taken to be only two years because the optimal strategy is often loss-leading. In the unconstrained model that we consider here, this can lead to an initially negative premium as $T$ is increased. The remaining parameters are fairly arbitrary, but they are chosen so that the initial premium is positive. Graphs of $f_{0}$ and the associated interior premium $k_{0}^{i}$ for $\gamma=0$ are shown in Figure 3. These results demonstrate that the interior premium strategy is optimal for this particular choice of parameters. For the parameters given 


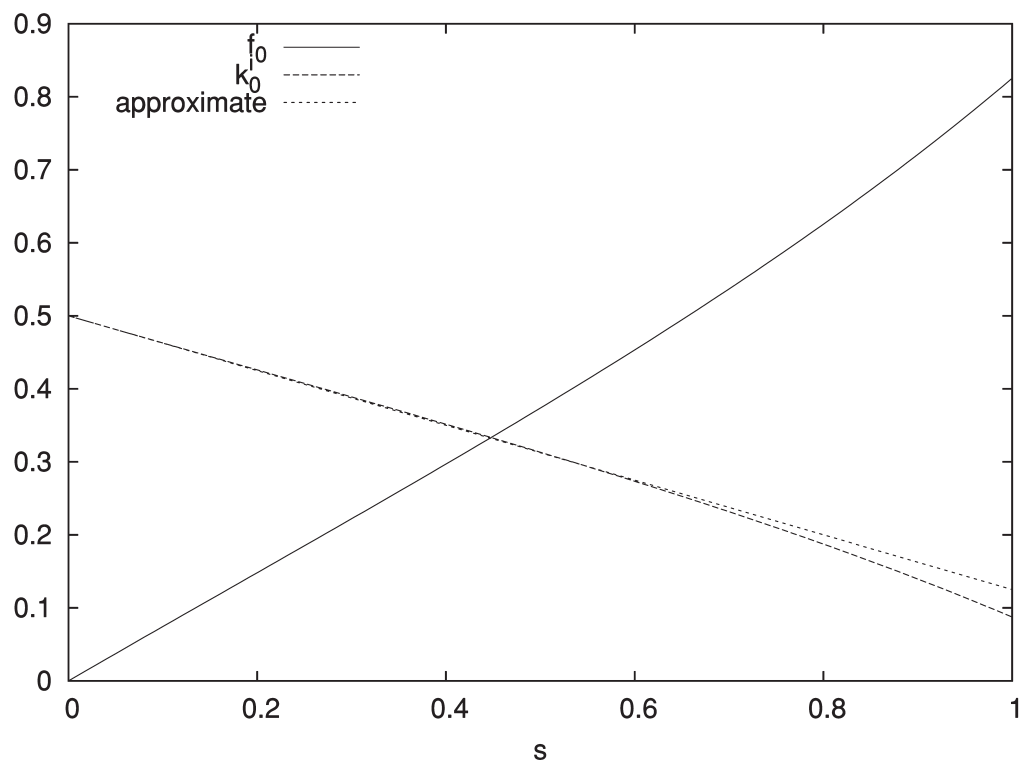

FIgURE 3: Optimal dynamic premium strategy for constant loss ratio $\gamma=0$ as a function of the time to termination $s$ using the parameters in Table 1 . The expression for $f_{0}$ is given by the first alternative in (33) and the approximate relative strategy is given by (23).

in Table 1 the first of the alternatives for $f_{0}$ is applicable. From (20) there is blowup if

$$
s_{b}=\frac{\pi-2 \tan ^{-1}\left(A_{0} / D_{0+}\right)}{D_{0+}} \in[0,1],
$$

and then $f_{0}\left(s_{b}\right)=\infty$. For these parameter sets the value function is infinite and it is possible to generate infinite wealth.

We adopt a simple explicit finite difference scheme to solve (31) with a first order time difference and second order spatial differences (Smith 1985). Consequently, there is a restriction on how large the time step can be in order to maintain numerical stability. In the computations, the nondimensional time step was taken as $\Delta s=10^{-3}$ and the spatial step was taken as $\Delta \gamma=10^{-2}$ unless stated otherwise. The results for the parameters in Table 1 are shown in Figure 4. Figure 4(a) shows the variation of the value function $V$ through (24) with the current state $\gamma_{t}=\gamma$. The largest value function occurs when $s=0$ and the loss ratio is $\gamma=0$ corresponding to, say, an infinite market average premium. From (29) and the boundary conditions (32) the interior relative premium is linear in $\gamma$ at termination $s=0$ and on $\gamma=b=1, k^{i}=b=1$ for this particular data set (see Figure 4(b)). 


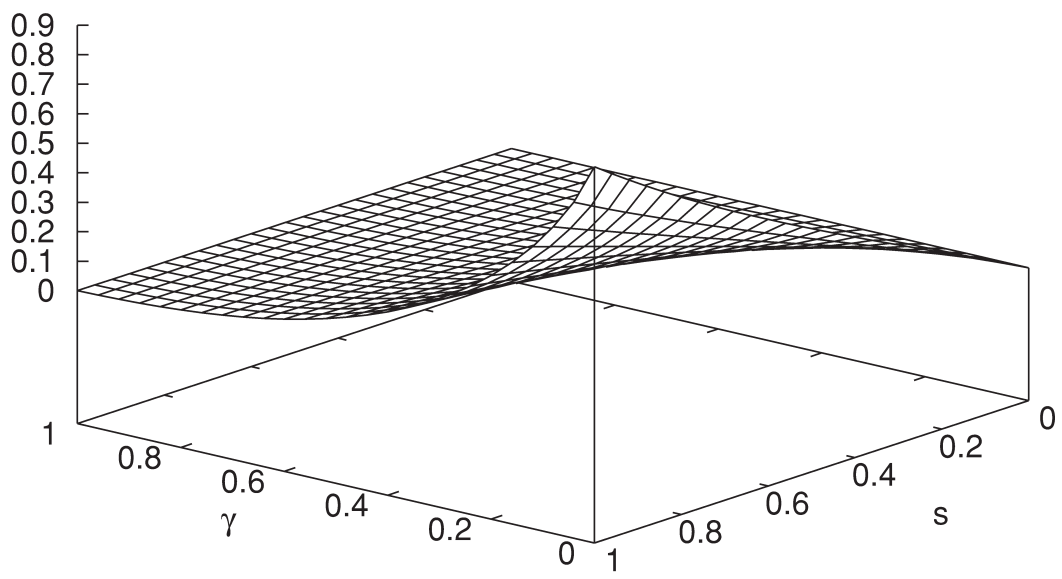

(a)

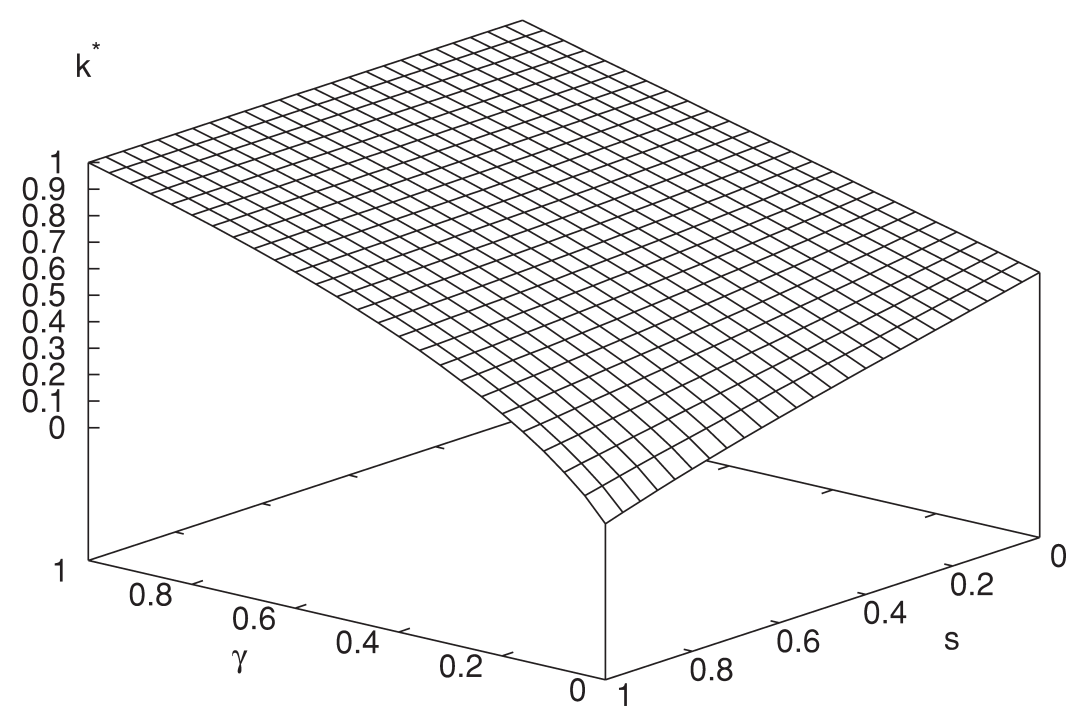

(b)

Figure 4: Optimal dynamic premium which maximises the expected terminal wealth using the parameters given in Table 1 . These parameters lead to a boundary condition with $f_{0}$ given by the first expression in

(33). Graph (a) shows the evolution of the function $f(s, \gamma)$ and graph (b) shows the optimal relative premium $k^{*}(s, \gamma)$. 
Suppose the finite difference grid is indexed by the points $\left\{(i, j) \mid i=0,1, \ldots, N_{s}\right.$; $\left.j=0,1, \ldots, N_{\gamma}\right\}$ where $N_{s}, N_{\gamma}$ are the number of time and spatial steps respectively. We adopt a fixed time step $\Delta s=1 / N_{s}$ and a fixed spatial step $\Delta \gamma=b / N_{\gamma}$ and on the grid we write $f_{i j}=f(i \Delta s, j \Delta \gamma)$. We can assess the convergence of the finite difference scheme by defining

$$
\|f\|=\frac{1}{\left(N_{s}+1\right)\left(N_{\gamma}+1\right)} \sum_{i, j}\left|f_{i j}\right|,
$$

which measures the size of $f$ over the domain and

$$
d g=\left(\Delta s^{2}+\Delta \gamma^{2}\right)^{1 / 2},
$$

which measures the resolution of the grid. In Figure 6 we plot $\|f\|$ against $d g$ using the grid refinement $N_{s}=10 k, N_{\gamma}=2000 k$ with $k=1,2, \ldots, 50$ and the parameters in Table 1 . It is clear that the scheme is converging to $\|f\| \sim 0.104$ as $d g \rightarrow 0$ with a linear convergence rate as one might expect given that we adopt a first order difference for the time step.

The optimal relative premium in Figure 4(b) gradually increases over the planning horizon and this increase is greatest when the loss ratio $\gamma=0$. As $\gamma=$ $\pi / \bar{p}$ decreases so the breakeven premium becomes much less than the market average premium $\bar{p}$. This means the insurance market is overpricing insurance and so there is greater scope to undercut the market price and still generate demand. This strategy leads to large market exposure through the demand law and large profits as can be seen in Figure 4(a) since as $f$ increases so does the value function $V$. The form of the premium strategy is strongly influenced by the qualitative features of the boundary condition on $\gamma=0$ given by (33). As the point of blow-up $s_{b}$ nears the domain $[0,1]$ so the values of $f_{0}$ become very large, which leads to negative values of $k_{0}$. If $k<\gamma$ then this is a loss-leading strategy so one can identify the first form of (33) as of loss-leading type providing the time horizon is sufficiently large.

We study the sensitivity of the model by varying the parameters $\rho, \sigma_{2}, a, b$, and $\phi$ in turn in Figures 5(a)-(e). The plots show the optimal relative premium $k^{*}$ at $t=0$ given the current state variable $\gamma$. The optimal strategy is fairly insensitive to the mean reversion, $\rho$, and the volatility of the loss ratio, $\sigma_{2}$. The expected value of the loss-ratio tends to one as $\rho$ gets large so that there are few opportunities for loss-leading and it is optimal to set a higher premium as shown in Figure 5(a). If the volatility is large and the current loss-ratio $\gamma$ is small then the loss ratio may substantially increase over the time horizon, so that it is optimal to set a higher premium and diminish loss-leading. Conversely, if the volatility and the current loss-ratio $\gamma$ are large then there is the possibility that the loss-ratio will substantially decrease over the planning horizon and lossleading opportunities will become available. This lowers the optimal premium as seen in Figure 5(b). 


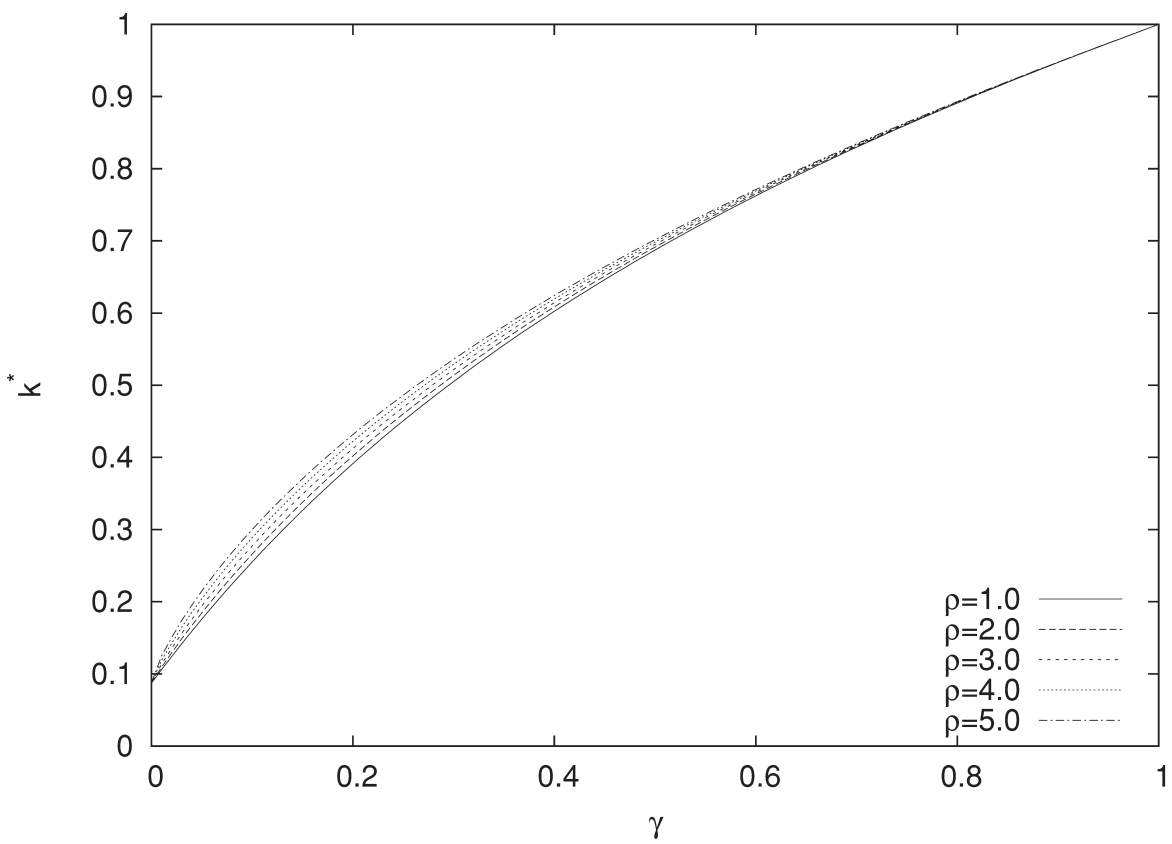

(a)

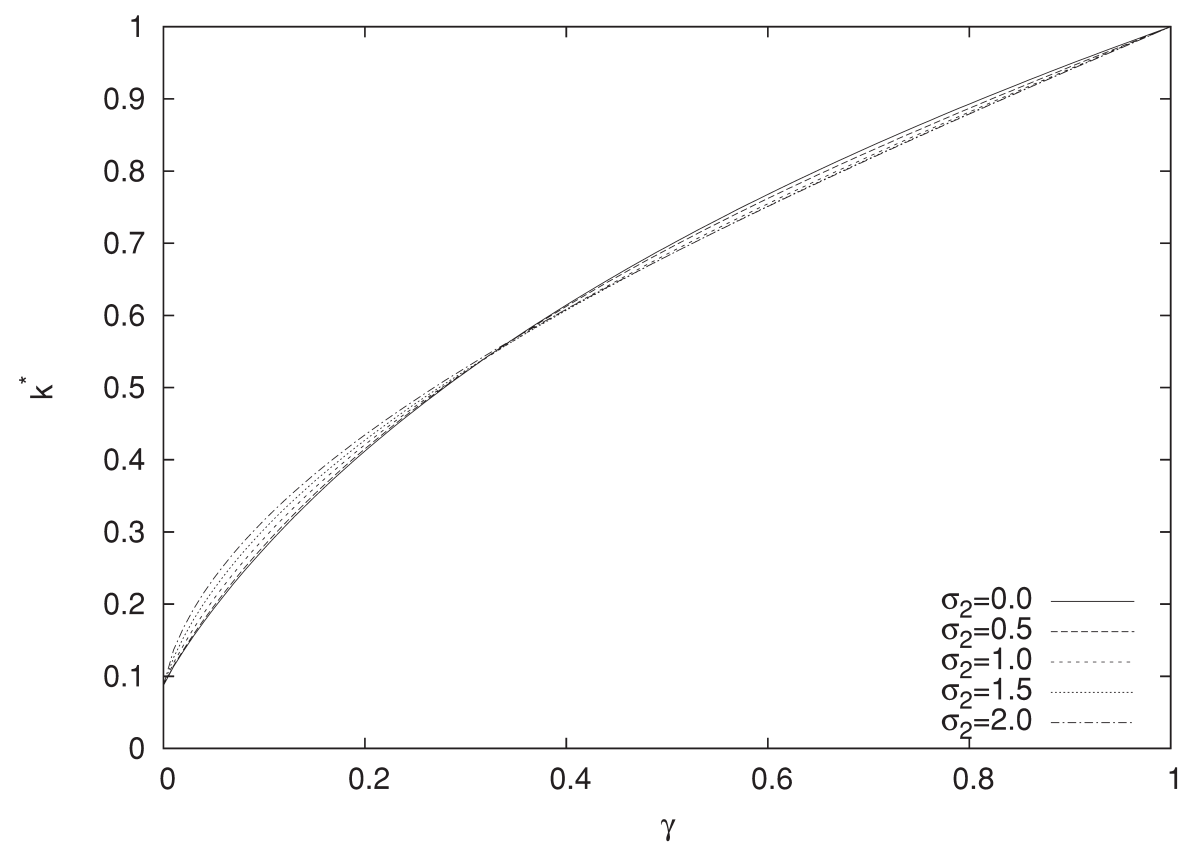

(b) 


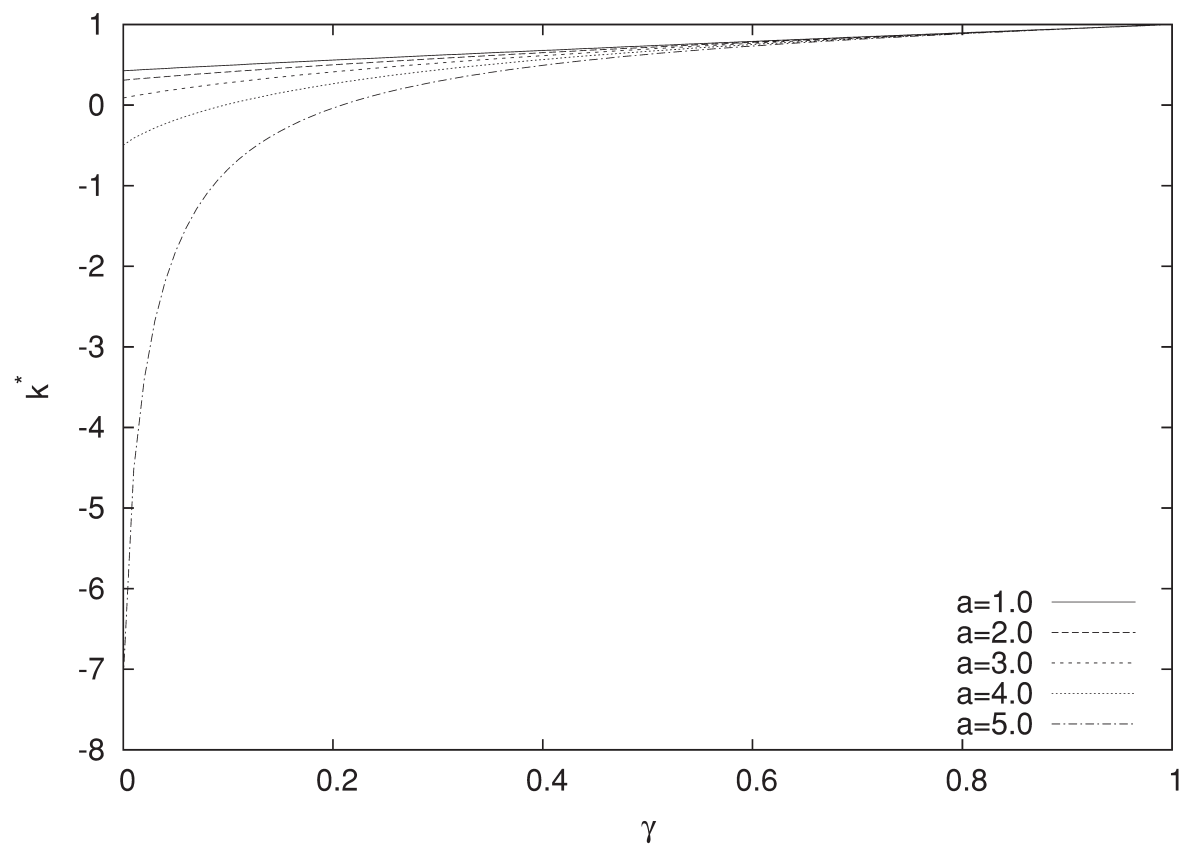

(c)

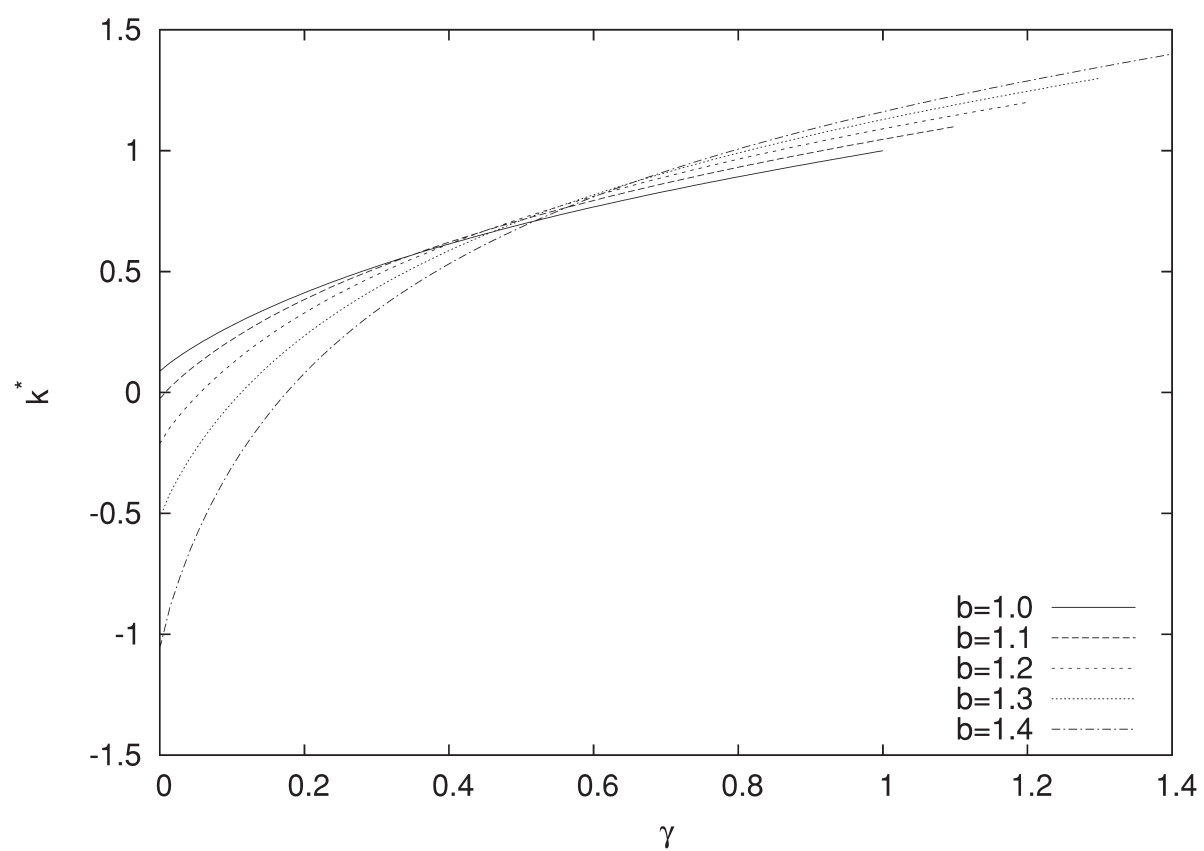

(d) 


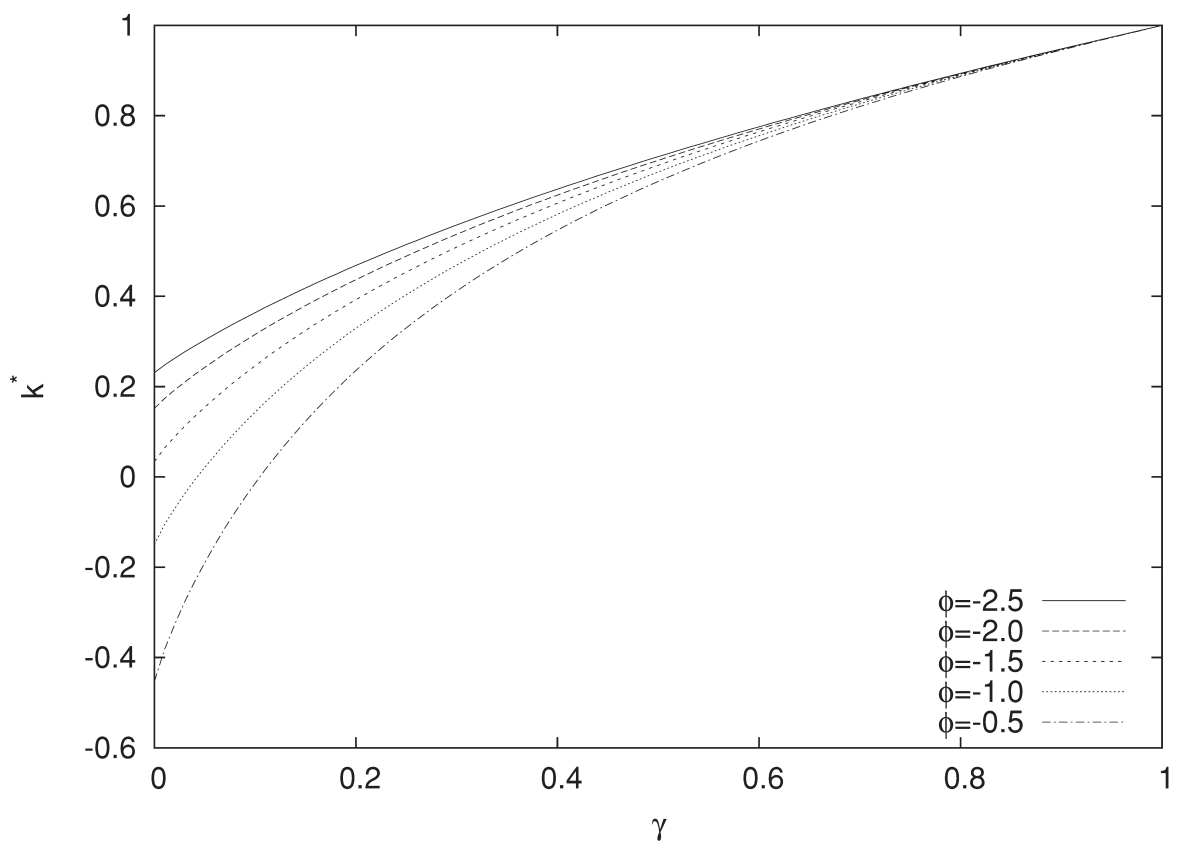

(e)

Figure 5: Sensitivity of the optimal initial relative premium $k^{*}(\gamma, 1)$ to (a) the loss ratio mean reversion $\rho$,

(b) the loss ratio volatility $\sigma_{2}\left(\Delta s=10^{-5}\right)$, (c) the demand parameter $a$, (d) the demand parameter $b$, and (e) the parameter $\phi$. The objective is to maximise the expected terminal wealth and the remaining data is taken from Table 1.

The optimal strategy is strongly dependent on the form of the demand function (parameters $a$ and $b$ ) and the parameter $\phi$ as shown in Figures 5(c)-(e). As $a$ or $b$ is increased the demand for insurance is larger for a fixed relative premium. This favours a loss-leading strategy because lowering the premium generates considerable exposure. Ultimately this leads to negative initial premium values as shown in Figure 5(c),(d) because there is no borrowing constraint in the model. The parameter $\phi$ encompasses the drift in the market average premium and the policy length: large growth and long policies tend to increase $\phi$, which increases the magnitude of loss-leading and leads to negative initial premium values. If the market average premium grows rapidly whilst the breakeven premium is fixed this again favours loss-leading.

\section{Total Discounted Utility of Wealth}

It is not clear that the objective of maximising the expected terminal wealth is the most appropriate goal for an insurer. Insurance companies must have 
sufficient funds to cover legal requirements and satisfy the demands of their shareholders. If an insurer adopts a strategy which leads to large losses in the expectation of significant income in the future then this generates substantial risk. An alternative objective often used in financial applications of stochastic control theory is to maximise the expected total discounted utility of wealth:

$$
\sup _{k}\left\{\int_{0}^{\mathcal{T}} e^{-\beta t} U\left(\mathbf{w}_{t}\right) d t\right\},
$$

where $U=U\left(\mathbf{w}_{t}\right)$ is the utility function and $\mathcal{T}=\tau \wedge T$. From such an objective one might expect a more conservative optimal strategy $k^{*}$ because initial lossleading does affect the objective. For simplicity, we consider the exponential utility function

$$
U(w)=\frac{1}{c}\left(1-e^{-c w}\right) \text { and } c>0 .
$$

This function has constant (absolute) risk aversion $c$ and is a member of the Hyperbolic Absolute Risk Aversion (HARA) family (Pratt 1964; Gerber \& Pafumi 1998). Browne (1995) used this utility function to calculate the optimal investment strategy for an insurer. We adopt a utility function of this form since as $c \rightarrow 0, U \rightarrow w$ so that the objective is linear in the wealth and it is easier to find the solution to the Bellman equation.

The appropriate definition for the value function is

$$
V(x, t)=\sup _{k} \mathbb{E}\left\{\int_{t}^{\mathcal{T}} e^{-\beta s} U\left(\mathbf{w}_{s}\right) d s \mid \mathbf{X}_{t}=x\right\},
$$

and the corresponding Bellman equation is

$$
\begin{array}{r}
V_{t}+\mu \bar{p} V_{\bar{p}}+\frac{1}{2} \sigma_{1}^{2} \bar{p}^{2} V_{\bar{p} \bar{p}}+\gamma\left(\frac{1}{2} \sigma_{2}^{2}-\rho \log \gamma\right) V_{\gamma}+\frac{1}{2} \sigma_{2}^{2} \gamma^{2} V_{\gamma \gamma}-\kappa q V_{q}-\alpha w V_{w}+ \\
q \sup _{k}\left\{G(k)\left(V_{q}+\bar{p}(k-\gamma) V_{w}\right)\right\}+\frac{e^{-\beta t}}{c}\left(1-e^{-c w}\right)=0,
\end{array}
$$

with boundary conditions

$$
V(\gamma=b, t)=0, \quad V(x, T)=0 .
$$

The interior premium is given by (10), as before, since the control does not enter the objective function in (35). Substituting the interior premium into the above equation yields

$$
\begin{aligned}
& V_{t}+\mu \bar{p} V_{\bar{p}}+\frac{1}{2} \sigma_{1}^{2} \bar{p}^{2} V_{\bar{p} \bar{p}}+\gamma\left(\frac{1}{2} \sigma_{2}^{2}-\rho \log \gamma\right) V_{\gamma}+\frac{1}{2} \sigma_{2}^{2} \gamma^{2} V_{\gamma \gamma}+ \\
& q\left(\frac{1}{2} a(b-\gamma)-\kappa\right) V_{q}+\left(-\alpha w+\frac{1}{4} a \bar{p} q(b-\gamma)^{2}\right) V_{w}+\frac{a q V_{q}^{2}}{4 \bar{p} V_{w}}+\frac{e^{-\beta t}}{c}\left(1-e^{-c w}\right)=0 .
\end{aligned}
$$


Again, we suppose that the first order condition does fulfill the maximisation operation in the Bellman equation and that the value function is sufficiently smooth that Ito's lemma can be applied in deriving (36).

At termination $t=T$ the optimal premium is undefined because the value function is identically zero. This should be contrasted with the well-defined terminal optimal premium given by (11) for the terminal wealth case. However, near the end of the time horizon at $t=T-\delta t$ where $\delta t$ is a small time increment we find by differencing the Bellman equation

$$
V_{T-\delta t}=\frac{\delta t e^{-\beta T}}{c}\left(1-e^{-c w}\right) .
$$

Therefore, $V_{q}=0$ and the terminal optimal premium is identical to (11) as $\delta t \rightarrow 0$ providing that the differencing is consistent with the spatial boundary conditions.

We nondimensionalise the Bellman equation by using the scales in (30) supplemented by

$$
\bar{p}=[\bar{p}] \hat{p}, q=[q] \hat{q}, w=[\bar{p}][q] \hat{w}, V=[\bar{p}][q] T, \hat{\beta}=\beta T,
$$

where $[\bar{p}]$ and $[q]$ are scales derived from the value of the market average premium and the exposure at $t=0$. Let us introduce the parameter

$$
\varepsilon=c[\bar{p}][q],
$$

as a dimensionless measure of the risk aversion of the insurer. Henceforth we shall drop the hats on the nondimensional variables. The nondimensional Bellman equation is therefore

$$
\begin{array}{r}
V_{s}=\mu \bar{p} V_{\bar{p}}+\frac{1}{2} \sigma_{1}^{2} \bar{p}^{2} V_{\bar{p} \bar{p}}+\gamma\left(\frac{1}{2} \sigma_{2}^{2}-\rho \log \gamma\right) V_{\gamma}+\frac{1}{2} \sigma_{2}^{2} \gamma^{2} V_{\gamma \gamma}-\kappa q V_{q}-\alpha w V_{w}+ \\
q \sup _{k}\left\{G(k)\left(V_{q}+\bar{p}(k-\gamma) V_{w}\right)\right\}+\frac{e^{-\beta(1-s)}}{\varepsilon}\left(1-e^{-\varepsilon w}\right) .
\end{array}
$$

We aim to solve this equation approximately using a perturbation expansion for $\varepsilon \ll 1$.

\subsection{No Risk Aversion}

If the insurer has no risk aversion then $\varepsilon=0$ (corresponding to $c \rightarrow 0$ ) and the utility function is linear in the wealth process. We look for a solution of the form

$$
V(x, s)=e^{-\beta(1-s)}(g(s) w+q \bar{p} h(\gamma, s)) .
$$


Substituting into (38) we obtain two equations for $g$ and $h$ :

$$
\begin{gathered}
g^{\prime}+(\alpha+\beta) g-1=0, \\
h_{s}=\gamma\left(\frac{1}{2} \sigma_{2}^{2}-\rho \log \gamma\right) h_{\gamma}+\frac{1}{2} \sigma_{2}^{2} \gamma^{2} h_{\gamma \gamma}+\sup _{k}\{G(k)(h+(k-\gamma) g)\},
\end{gathered}
$$

where the boundary conditions are derived in a similar way to the terminal wealth case and are

$$
g(0)=0, h(0, s)-h_{0}(s)=h(b, s)=h(\gamma, 0)=0 .
$$

In terms of these functions the interior relative premium is

$$
k^{i}=\frac{1}{2}\left(b+\gamma-\frac{h(\gamma, s)}{g(s)}\right)
$$

and when the control is interior the equation for $h$ becomes

$$
h_{s}=\gamma\left(\frac{1}{2} \sigma_{2}^{2}-\rho \log \gamma\right) h_{\gamma}+\frac{1}{2} \sigma_{2}^{2} \gamma^{2} h_{\gamma \gamma}+C(s) h^{2}+D(\gamma) h+E(\gamma, s),
$$

where

$$
\begin{aligned}
C(s) & =\frac{a}{4 g(s)}, \\
D(\gamma) & =\frac{1}{2} a(b-\gamma)+\mu-\beta-\kappa, \\
E(\gamma, s) & =\frac{1}{4} a(b-\gamma)^{2} g(s) .
\end{aligned}
$$

The equation for $g$ can be integrated immediately to give

$$
g(s)=\frac{1}{\alpha+\beta}\left(1-e^{-(\alpha+\beta) s}\right),
$$

and, as before, the function $h_{0}$ is given by the solution to the problem independent of $\gamma$ :

$$
\frac{d h_{0}}{d s}=C(s) h_{0}^{2}+D(0) h_{0}+E(0, s)
$$

with terminal condition $h_{0}(0)=0$. This is a Riccati equation for which there is no general technique for obtaining a solution. Moreover, these equations can exhibit spontaneous singularities for finite $s$ : this phenomenon restricts the parameter range over which the control will be smooth in a similar way to (17).

In order to determine how the relative premium differs from Section 3 we set

$$
\mathcal{F}(\gamma, s)=\frac{h(\gamma, s)}{g(s)},
$$




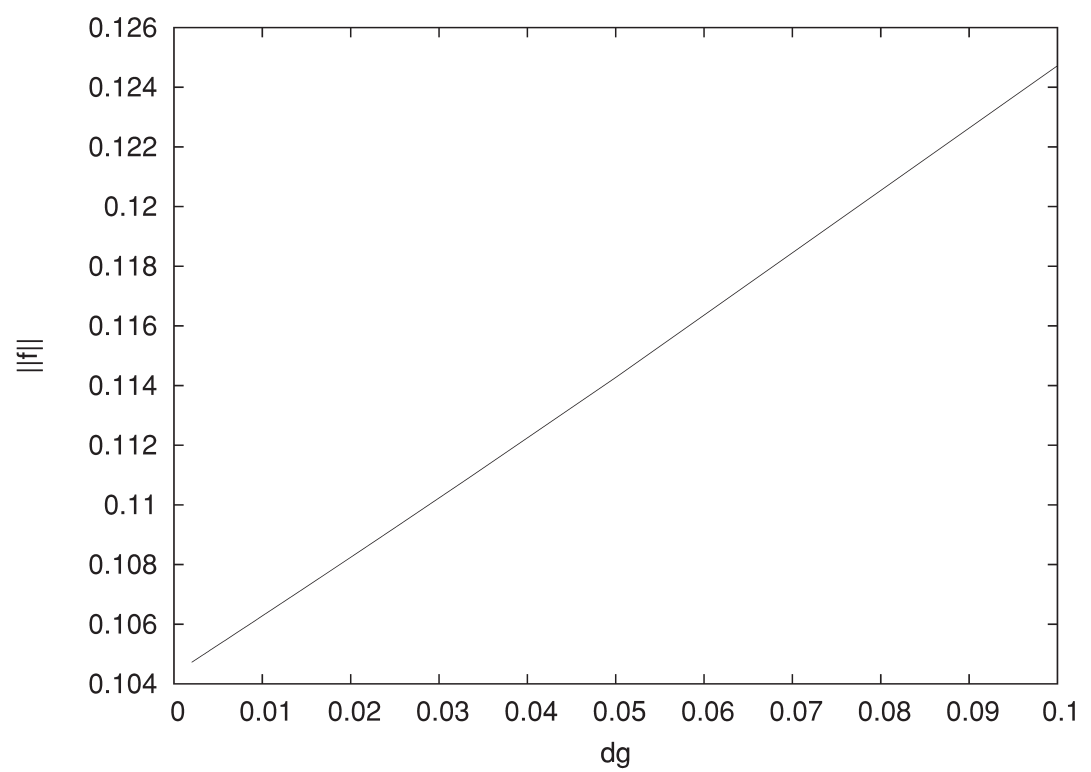

FIGURE 6: Convergence of the finite difference scheme as the mesh is refined. The resolution of the mesh is proportional to $d g$ while $\|f\|$ is the average value of $f$ over the domain.

so that (29) and (43) have the same form. After some manipulation (44) becomes

$$
\mathcal{F}_{s}=\gamma\left(\frac{1}{2} \sigma_{2}^{2}-\rho \log \gamma\right) \mathcal{F}_{\gamma}+\frac{1}{2} \sigma_{2}^{2} \gamma^{2} \mathcal{F}_{\gamma \gamma}+\frac{1}{4} a \mathcal{F}^{2}+\left(A(\gamma)-\frac{1}{g(s)}\right) \mathcal{F}+B(\gamma) \text {. }
$$

Notice there is an extra term $-\mathcal{F} / g$ on the RHS of this equation compared with (27). Since $g(s)$ is a non-negative function and we expect $\mathcal{F}$ is positive as before, the gradient $\mathcal{F}_{s}$ is reduced and so $-\mathcal{F}$ is increased as the time to termination, $s$, is increased. Therefore, in comparison with the terminal wealth case, the optimal relative premium is larger and the strategy is more conservative. If the discount rate $\beta$ is made larger then $g$ decreases and so $\mathcal{F}$ is made smaller. This in turn makes $-\mathcal{F}$ larger and therefore increases the interior premium $k^{i}$.

We can reduce the free parameter set by writing

$$
\zeta=\alpha+\beta \text {. }
$$

Thus the nondimensional free parameters are $\rho, \sigma_{2}, a, b, \phi, \zeta:$ these parameters and the current state, $x$, determine the optimal relative premium. We have an additional free parameter over the terminal wealth case because we have introduced the discount factor $\beta$.

Again, an explicit finite difference scheme is used to solve equation (41) with the boundary conditions in (42) using the grid $\Delta s=10^{-4}, \Delta \gamma=10^{-2}$. Figure 7 


\section{h}

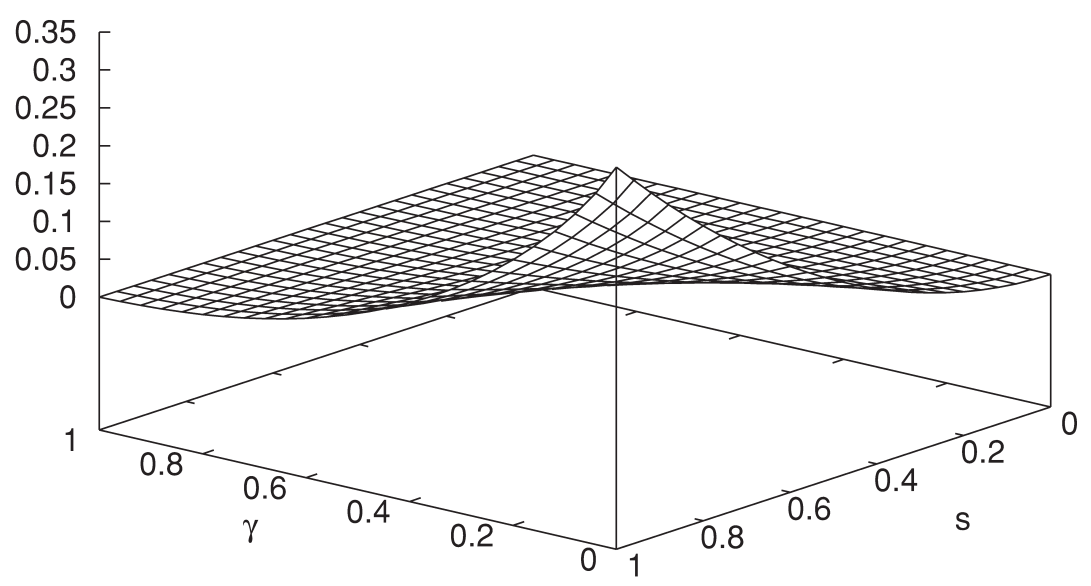

(a)

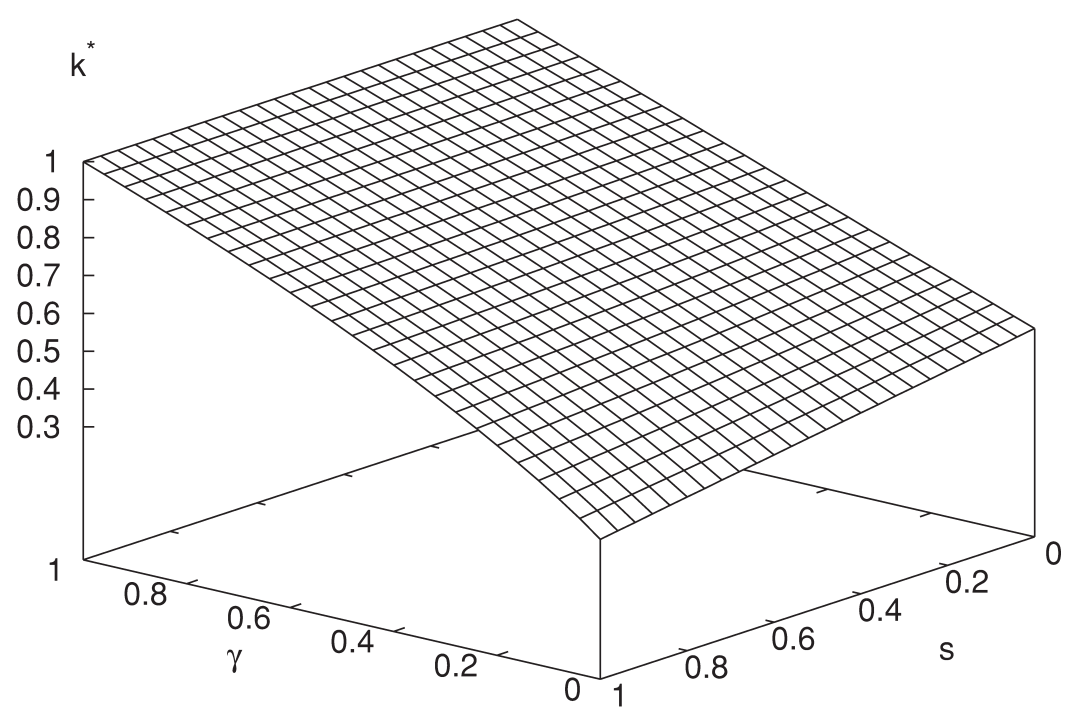

(b)

FIGURE 7: Optimal dynamic premium which maximises the total discounted utility of wealth using the parameters given in Table 1 with a linear utility function. Graph (a) shows the evolution of the function $h(s, \gamma)$ and graph (b) shows the optimal relative premium $k^{*}(s, \gamma)$. 
shows plots of $h(\gamma, s)$ and $k^{*}(\gamma, s)$ for the sample data set in Table 1 . The optimal strategy is qualitatively similar to the terminal wealth case although the optimal relative premium is larger. The optimal premium strategy is more conservative here because loss-leading affects the total utility of wealth of the insurer. The robustness of the control with respect to the objective gives us confidence that the analytical control in (14) is an appropriate strategy to consider for an insurer. The sensitivity of the optimal control to the model parameters is similar to the terminal wealth case and so this is not shown.

\subsection{Weakly Nonlinear Utility Function}

We consider a nonlinear objective by including the next term in an asymptotic expansion for the utility function

$$
U(w) \sim w-\frac{1}{2} \varepsilon w^{2},
$$

where we assume $w \sim O(1)$ by the choice of scaling.

We use a perturbation expansion for $V$ in terms of $\varepsilon \ll 1$ to yield a succession of linear systems. Thus we write

$$
\begin{aligned}
& V \sim V_{0}+\varepsilon V_{1}+\ldots \\
& k^{*} \sim k_{0}^{*}+\varepsilon k_{1}^{*}+\ldots
\end{aligned}
$$

Substituting into (38) and assuming an interior control, $V_{0}$ is given by (39) while at $O(\varepsilon)$ we find

$$
\begin{gathered}
V_{1 s}=\mu \bar{p} V_{1 \bar{p}}+\frac{1}{2} \sigma_{1}^{2} \bar{p}^{2} V_{1 \bar{p} \bar{p}}+\gamma\left(\frac{1}{2} \sigma_{2}^{2}-\rho \log \gamma\right) V_{1 \gamma}+\frac{1}{2} \sigma_{2}^{2} \gamma^{2} V_{1 \gamma \gamma}+ \\
q\left(\frac{1}{2} a(b-\gamma)-\kappa\right) V_{1 q}+\left(-\alpha w+\frac{1}{4} a \bar{p} q(b-\gamma)^{2}\right) V_{1 w}+ \\
\frac{a q h(\gamma, t)}{4 g(t)}\left(2 V_{1 q}-\bar{p} h \frac{V_{1 w}}{g(t)}\right)-\frac{1}{2} e^{-\beta(1-s)} w^{2},
\end{gathered}
$$

and

$$
V_{1}(\gamma=b, s)=0, \quad V_{1}(x, 0)=0
$$

This is a linear PDE with four space dimensions. The value function $V_{1}$ is not linear in each of the state variables because of the last term in the equation.

We aim to reduce the dimension of the problem by looking for a solution of the form

$$
V_{1}(x, s)=e^{-\beta(1-s)}\left(m(\gamma, s) \bar{p}^{2} q^{2}+n(\gamma, s) \bar{p} q w+r(s) w^{2}\right) .
$$


Substituting (50) into (49) and collecting together the $O(1), O(w)$ and $O\left(w^{2}\right)$ terms yields three equations for $m, n$ and $r$ :

$$
\begin{aligned}
m_{s}=\gamma\left(\frac{1}{2} \sigma_{2}^{2}-\rho \log \gamma\right) m_{\gamma}+\frac{1}{2} \sigma_{2}^{2} \gamma^{2} m_{\gamma \gamma}+ & \\
& \left(a(b-\gamma)+2 \phi-\alpha-\zeta+\sigma_{1}^{2}+\frac{a h}{g}\right) m+\frac{a}{4}\left((b-\gamma)^{2}-\frac{h^{2}}{g^{2}}\right) n, \\
n_{s}=\gamma\left(\frac{1}{2} \sigma_{2}^{2}-\rho \log \gamma\right) n_{\gamma}+\frac{1}{2} \sigma_{2}^{2} \gamma^{2} n_{\gamma \gamma}+ & \\
& \left(\frac{1}{2} a(b-\gamma)+\phi-\alpha-\zeta+\frac{a h}{2 g}\right) n+\frac{a}{2}\left((b-\gamma)^{2}-\frac{h^{2}}{g^{2}}\right) r, \\
& \frac{d r}{d s}=-(2 \alpha+\beta) r-\frac{1}{2},
\end{aligned}
$$

with boundary conditions

$$
\begin{gathered}
m(0, s)-m_{0}(s)=m(b, s)=m(\gamma, 0)=0, \\
n(0, s)-n_{0}(s)=n(b, s)=n(\gamma, 0)=0, \\
r(0)=0,
\end{gathered}
$$

and where $g=g(s)$ and $h=h(\gamma, s)$ are given by (41) and (45). The boundary functions $m_{0}(s)$ and $n_{0}(s)$ satisfy the coupled ODEs:

$$
\begin{aligned}
& \frac{d m_{0}}{d s}=\left(a b+2 \phi-\alpha-\zeta+\sigma_{1}^{2}+\frac{a h_{0}}{g}\right) m_{0}+\frac{a}{4}\left(b^{2}-\frac{h_{0}^{2}}{g^{2}}\right) n_{0}, \\
& \frac{d n_{0}}{d s}=\left(\frac{1}{2} a b+\phi-\alpha-\zeta+\frac{a h_{0}}{2 g}\right) n_{0}+\frac{a}{2}\left(b^{2}-\frac{h_{0}^{2}}{g^{2}}\right) r,
\end{aligned}
$$

with initial conditions $m_{0}(0)=n_{0}(0)=0$. On integrating (51) and applying the boundary condition we obtain

$$
r(s)=\frac{e^{-(2 \alpha+\beta) s}-1}{2(2 \alpha+\beta)} .
$$

From (10) the leading-order interior relative premium is given by (43) whilst the second-order term is

$$
\begin{aligned}
k_{1}^{*} & =\frac{V_{0 q}}{2 \bar{p} V_{0 w}}\left(\frac{V_{1 w}}{V_{0 w}}-\frac{V_{1 q}}{V_{0 q}}\right) \\
& =\frac{1}{2 g(s)}\left(\frac{h(\gamma, s)}{g(s)}(\bar{p} q n(\gamma, s)+2 r(s) w)-2 q \bar{p} m(\gamma, s)-w n(\gamma, s)\right) .
\end{aligned}
$$


For a nonlinear objective function the optimal relative premium is a function of all four state variables. This complicates the comparison of how the premium strategy changes if the insurer changes its objective. Also note that $m(\gamma, s)$ depends on the coefficient of volatility of the market average premium, $\sigma_{1}$, and consequently so does the relative optimal premium.

Figure 8(a) and (b) show the solutions for $m$ and $n$ computed using an explicit finite difference scheme and the parameters in Table 1 . All of the functions $m, n$ and $r$ are negative over the domain of integration indicating that risk aversion decreases the value function $V$. The greatest decrease in $V$ is at $t=\gamma=0$ since that is where most wealth is generated. Figure 8(c) shows the perturbation to the optimal relative premium if we set the current state variables $\bar{p} \equiv q \equiv w \equiv 1$. Notice that $k_{1}^{*}$ is positive for these processes, so that increasing the risk aversion increases the optimal relative premium. This increase is greatest if the current loss ratio is small. Figure 9 shows the qualitative effect of increasing the insurer's risk aversion $\varepsilon$. Risk aversion leads to a more conservative interior premium strategy - this generates less exposure over the planning horizon, which decreases the total claims and maintains premium income.

\section{CONCLUSions}

We have found the optimal premium strategy for an insurer in a competitive market using optimal control theory. In general, the Bellman equation arising from control theory contains a degenerate diffusion operator. For the current model we have shown how this degeneracy can be removed by a change of variables, which makes the resulting problems easy to solve numerically. The choice of a linear demand function (in the relative premium) leads to a single nonlinear term in the Bellman equation which considerably simplifies the analysis. Our ability to find the optimal premium strategy is limited by the values given to the model parameters. We have found the conditions that lead to a positive initial premium for the terminal wealth case. In general, if the optimal control is smooth then the optimal premium gradually increases over the term of the planning horizon for the parameter sets considered here. We find that as risk aversion is increased so does the optimal relative premium strategy. This generates lower exposure and so ultimately lower overall wealth. The numerical value of the terminal relative premium is identical for all the objectives studied here.

One significant assumption is that we can treat the market distinctly from the insurer so that whatever the insurer's premium, the market does not react with a competitive price. Currently we are investigating how one might relax this assumption by incorporating an adjustment term in the process followed by the market average premium. Another modelling simplification is the specification of a stochastic process for the breakeven premium. This premium represents the cost of insurance for the insurer and assessment of this quantity requires a good model for the claims process and an accurate definition of the loading 


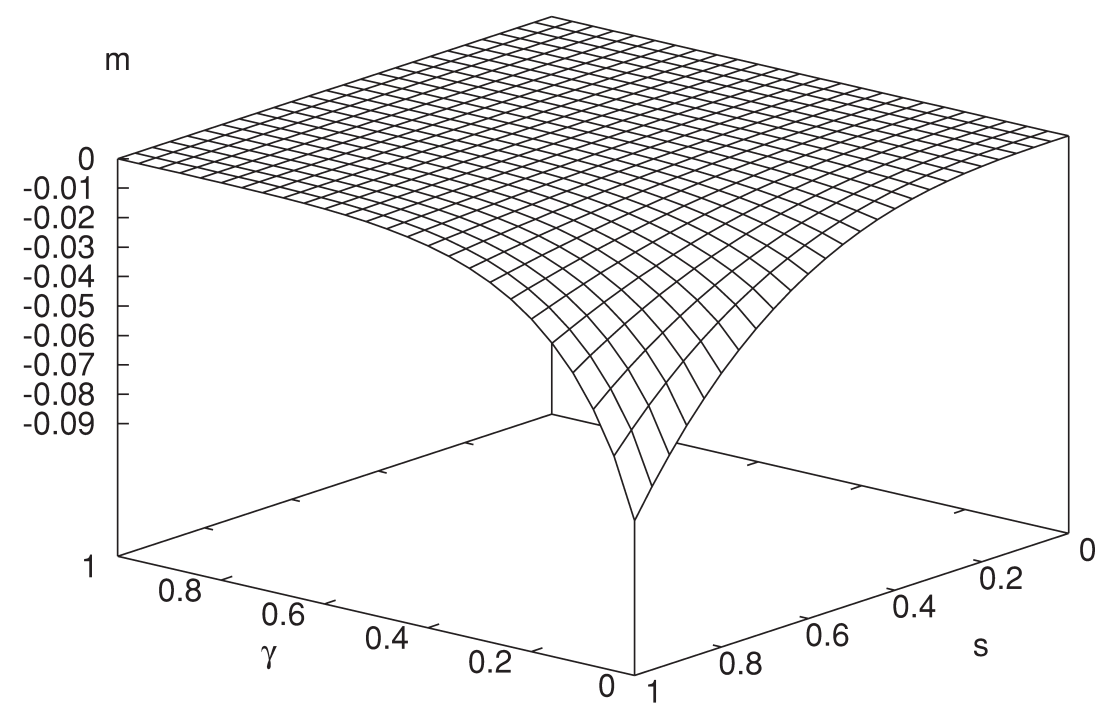

(a)

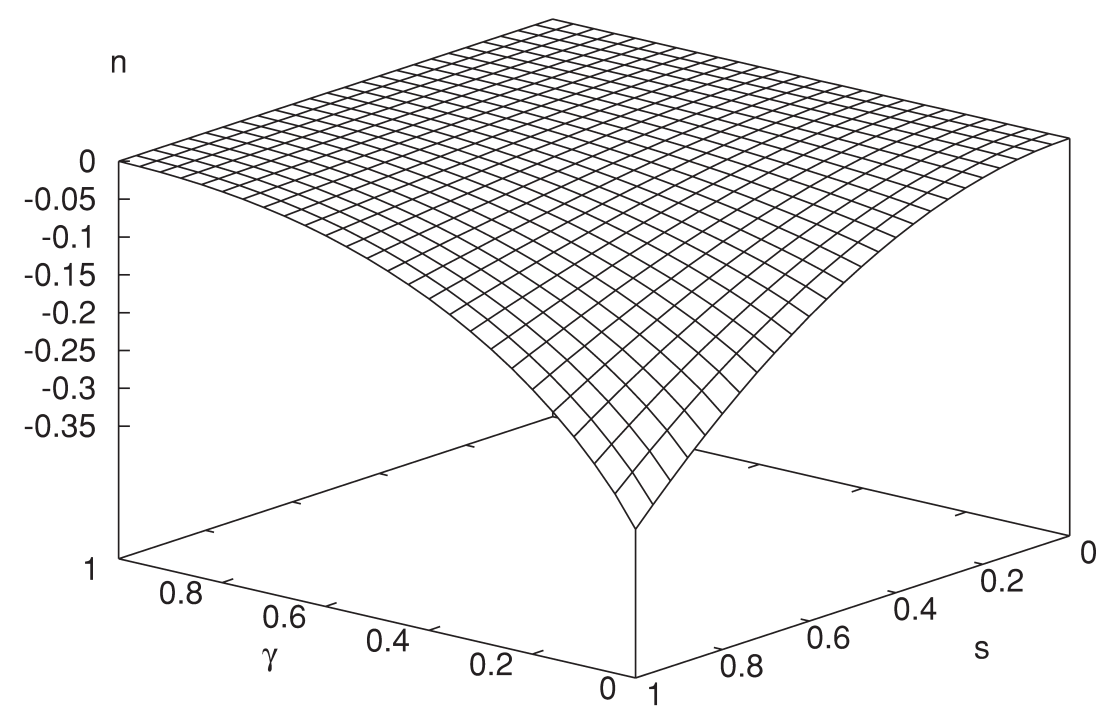

(b) 


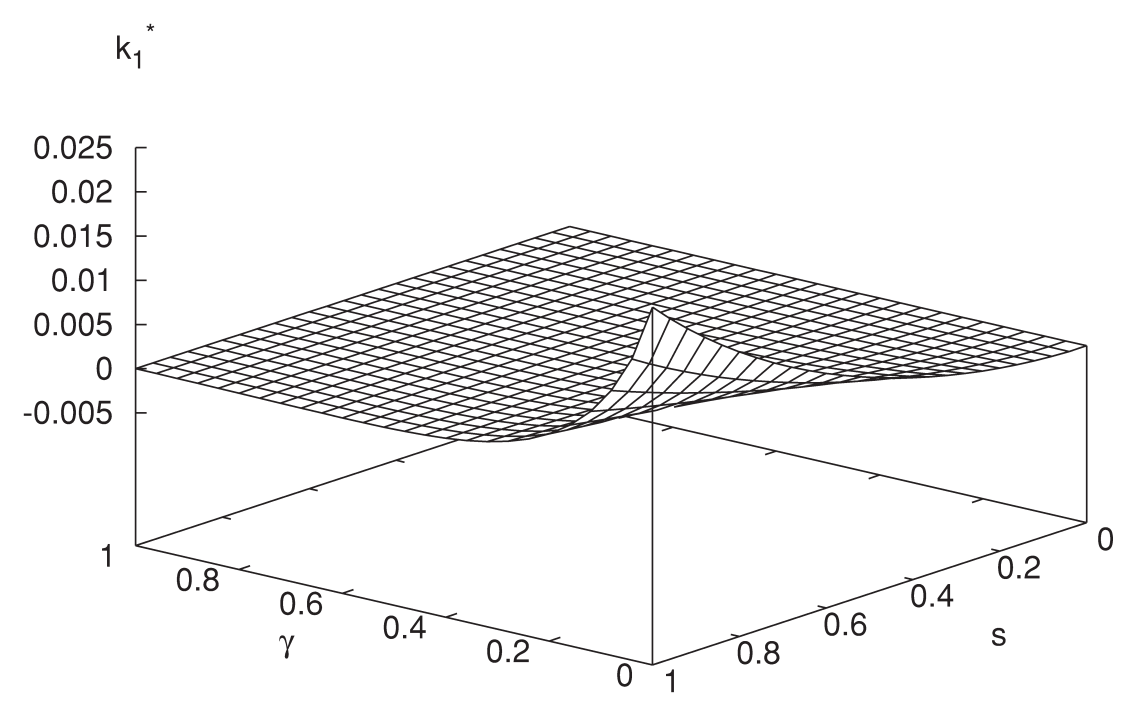

(c)

Figure 8: Optimal premium strategy when the insurer maximises its expected total utility of wealth.

The utility function is a quadratic function of the wealth $w$ in (48) and represents the second order expansion of an exponential utility function when risk aversion is small. Plots (a) and (b) show the solution for $m(\gamma, s)$ and $n(\gamma, s)$ for the parameters in Table 1, while (c) shows the deviation in the interior relative premium $k_{1}^{*}(\gamma, s)$ for $\bar{p}=q=w=1$.

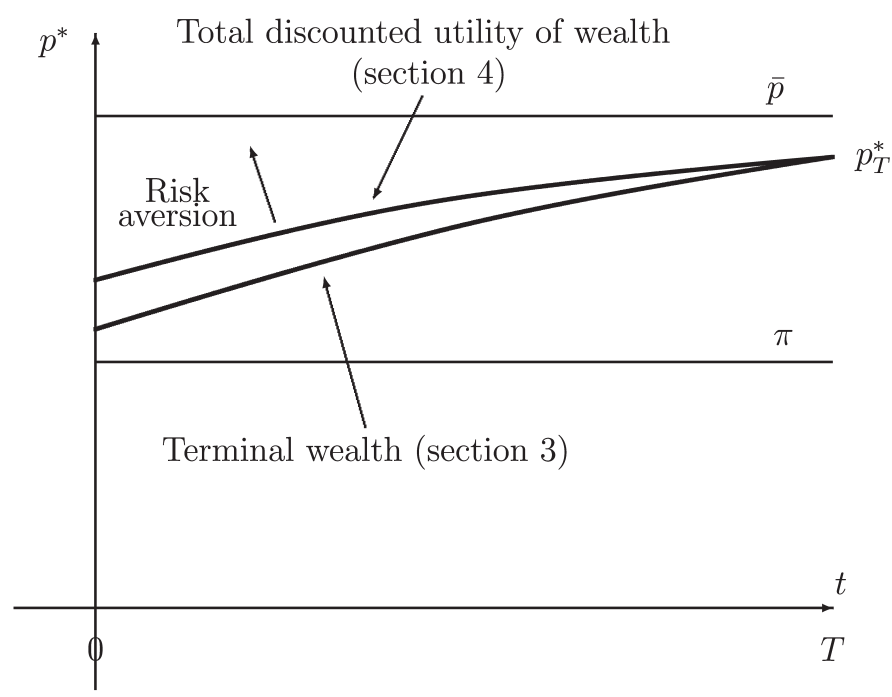

Figure 9: Qualitative comparison of the optimal premium strategy $p^{*}$ if the loss-ratio is constant and the market average premium is constant for the objective of either maximising expected terminal wealth or total discounted utility of wealth with a linear utility function. The loss ratio in the diagram is $\gamma=1 / 2$ so the terminal optimal premium is $p_{T}^{*}=\frac{1}{2} \bar{p}\left(b+\frac{1}{2}\right)$. 
factor. The benefit of using this process to define a loss-ratio is that the wealth process $\mathbf{w}_{t}$ directly reflects the current wealth of the insurer including its liabilities.

Sample paths have been restricted to prevent the insurer leaving and the reentering the insurance market. If one relaxes this assumption then one must cope with the non-differentiability of the demand function (9) at $k=b$ and the determination of when the insurer should stop selling insurance. We have introduced this non-differentiability in order that the feedback control is tractable. However, it is not clear that there is a classical solution to the Bellman equation for the more general problem and so one cannot apply a verification theorem to ensure optimality of the feedback control. The problem is reminiscent of the American option pricing problem posed as an optimal control problem from which one obtains a semi-linear Black-Scholes equation with a discontinuous cash flow. Benth, Karlson \& Reikvam (2003) have determined simple numerical schemes using viscosity solutions of the Black-Scholes equation (Fleming \& Soner 1993). For these schemes it is not required to track the free boundary representing when it is optimal to exercise the option: its position is given implicitly from the numerical solution. It is tempting to suggest that a similar construction might be applied to the competitive pricing problem when the insurer is permitted to have periods of no sales.

The difficulty with using dynamic programming to calculate an optimal premium strategy lies with the balance between posing a realistic model and the ability to solve and interpret the resulting high-dimensional Bellman equation. We have found an analytical solution for a restricted case, and shown that the numerical solution of the more general problem is feasible, even for a quite sophisticated objective function. Often the premium that the insurer can set is constrained by both legislation and the desire to minimise the risk of loss-leading. The incorporation of constraints into the optimal control problem is another area of ongoing research.

\section{ACKNOWLEDGEMENTS}

We gratefully acknowledge the financial support of the EPSRC under grant GR/S23056/01 and the Actuarial Research Club of the Cass Business School, City University.

\section{APPENDIX}

\section{A. Mean Claim Size Rate Process}

If we drop the assumption that the evolution of the breakeven premium can be deduced from policy data then we must deal directly with the mean claim size rate (and related claims expenses) $\mathbf{u}_{t}$. This is, for example, the average size of claims received in one day per unit of exposure. It must be measured per 
unit time because it incorporates the intensity of claims as well as their magnitude. An appropriate wealth process is now given by

$$
d \mathbf{w}_{t}=-\alpha \mathbf{w}_{t} d t+\mathbf{p}_{t} \mathbf{b}_{t} d t-\mathbf{u}_{t} \mathbf{q}_{t} d t .
$$

As before, the new and renewed business generates wealth $\mathbf{p}_{t} \mathbf{b}_{t} \delta t$ in time $\delta t$ while claims are now accounted for as they arise so that the loss of wealth is $\mathbf{u}_{t} \mathbf{q}_{t} \delta t$.

In order to calculate a simple expression for the liabilities, it is convenient to adopt separate processes for the market average premium and the mean claim size rate. We suppose that the market average premium reverts to current cost of insurance in the long term and that the mean claim size rate is lognormally distributed:

$$
\begin{aligned}
d \overline{\mathbf{p}}_{t} & =\lambda\left(\eta \mathbf{u}_{t}-\overline{\mathbf{p}}_{t}\right) d t+\mu_{1} \overline{\mathbf{p}}_{t} d t+\sigma_{1} \overline{\mathbf{p}}_{t} d \mathbf{W}_{1 t}, \\
d \mathbf{u}_{t} & =\mathbf{u}_{t}\left(\mu_{2} d t+\sigma_{2} d \mathbf{W}_{2 t}\right),
\end{aligned}
$$

where the mean reversion rate is $\lambda$. The drifts $\mu_{1}, \mu_{2}$ and coefficients of volatility $\sigma_{1}, \sigma_{2}$ are taken as constants. The current cost of insurance per unit exposure for the whole market is

$$
\mathbb{E}\left[\int_{t}^{t+\tau} \mathbf{u}_{s} d s \mid \mathbf{X}_{t}\right]=\eta u_{t},
$$

where $\tau$ is the length of policies, the current state of the system is $\mathbf{X}_{t}=\left(\overline{\mathbf{p}}_{t}, \mathbf{u}_{t}\right.$, $\left.\mathbf{q}_{t}, \mathbf{w}_{t}\right)$ and

$$
\eta=\left(\frac{e^{\mu_{2} \tau}-1}{\mu_{2}}\right) .
$$

If no more insurance is sold at time $t$ then the outstanding claims generate a loss of wealth for the insurer equal to

$$
\int_{t}^{\infty} \mathbf{u}_{s} \mathbf{q}_{s} d s
$$

and the exposure decays exponentially according to (5):

$$
q_{s}=q_{t} e^{-\kappa(s-t)},
$$

for $s \geq t$. The expected liabilities of the insurer at the end of the time horizon is therefore

$$
\mathbb{E}\left[q_{T} e^{\kappa T} \int_{T}^{\infty} \mathbf{u}_{s} e^{-\kappa s} d s \mid \mathbf{X}_{T}\right]=\frac{q_{T} u_{T}}{\kappa-\mu_{2}} .
$$

We must have $\kappa>\mu_{2}$ otherwise the outstanding liabilities are infinite. 
If the insurer aims to maximise its net terminal wealth then the appropriate value function is

$$
V(x, t)=\sup _{p} \mathbb{E}\left[\mathbf{w}_{T}-\frac{\mathbf{q}_{T} \mathbf{u}_{T}}{\kappa-\mu_{2}} \mid \mathbf{X}_{t}=x\right] .
$$

Assuming the value function is sufficiently smooth the corresponding Bellman equation is

$$
\begin{gathered}
V_{t}+(\lambda(\eta u-\bar{p})+\mu \bar{p}) V_{\bar{p}}+\frac{1}{2} \sigma_{1}^{2} \bar{p}^{2} V_{\bar{p} \bar{p}}+\mu_{2} u V_{u}+\frac{1}{2} \sigma_{2}^{2} u^{2} V_{u u}+ \\
\sup _{p}\left\{q(G-\kappa) V_{q}+(-\alpha w+q(G p-u)) V_{w}\right\}=0,
\end{gathered}
$$

with boundary condition

$$
V(t=T)=w-\frac{q u}{\kappa-\mu_{2}} .
$$

The first order condition for a maximum gives the interior relative premium as

$$
k^{i}=\frac{1}{2}\left(b-\frac{V_{q}}{\bar{p} V_{w}}\right)
$$

where we have adopted the linear demand function (9). Consequently at termination of the planning horizon this relative premium is

$$
k_{T}^{i}=\frac{1}{2}\left(b+\frac{u}{\bar{p}\left(\kappa-\mu_{2}\right)}\right) \text {. }
$$

If we write the loss ratio for this model using the conventional definition then $\gamma=\eta u / \bar{p}$. The terminal relative premium then only differs from (11) by the term $\eta\left(\kappa-\mu_{2}\right)$. For policies of length one year $\tau=1$ yr and $\mu_{2}=0.1 \mathrm{p}$.a. gives $\eta\left(\kappa-\mu_{2}\right) \sim 0.9$ so that the optimal strategies are similar near termination.

Let us suppose the value function is of the form

$$
V(x, t)=e^{\alpha(T-t)}(w+q f(\bar{p}, u, t)) .
$$

Substituting this function into (54) yields

$$
\begin{gathered}
f_{t}+\left(\lambda(\eta u-\bar{p})+\mu_{1} \bar{p}\right) f_{\bar{p}}+\frac{1}{2} \sigma_{1}^{2} \bar{p}^{2} f_{\bar{p} \bar{p}}+\mu_{2} u f_{u}+\frac{1}{2} \sigma_{2}^{2} u^{2} f_{u u}+ \\
\frac{a}{4 \bar{p}} f^{2}+\left(\frac{1}{2} a b-\kappa-\alpha\right) f+\frac{1}{4} a b^{2} \bar{p}-u=0,
\end{gathered}
$$

with the interior relative premium given by

$$
k^{i}=\frac{1}{2}\left(b-\frac{f(\bar{p}, u, t)}{\bar{p}}\right) .
$$


The function $f$ is not linear in $\bar{p}$ or $u$ because of the quadratic term in $f$ so it appears as though we must solve this equation numerically. Consequently, rather than present a premium strategy as a function of three independent variables $(t, \bar{p}$ and $u)$, we have chosen in the main body of the paper to model the breakeven premium directly, which yields a strategy which is simpler to interpret. However, the mean claim size rate model does have the merit of explicitly accounting for the risk in holding an exposure $\mathbf{q}_{t}$, and therefore may be a suitable model for studying the optimisation problem when there are control and state constraints.

If we drop (52) and explicitly relate the market average premium $\overline{\mathbf{p}}_{t}$ to the mean claim size rate $\mathbf{u}_{t}$ then the model only contains a single factor $\mathbf{W}_{2 t}$. In this case analytical solutions similar to (17) can be found.

\section{REFERENCES}

Asmussen, S. and TAKSAR, M. (1997) Controlled diffusion models for optimal dividend pay-out. Insurance: Mathematics and Economics, 20, 1-15.

Benth, F.E., Karlsen, K.H. and Reikvam, K. (2003) A semilinear Black and Scholes partial differential equation for valuing American options. Finance and Stochastics, 7, $277-298$.

Britton, N.F. (1986) Reaction-Diffusion Equations and Their Applications to Biology. Academic Press.

BRowne, S. (1995) Optimal investment policies for a firm with a random risk process: Exponential utility and minimizing the probability of ruin. Math. Oper. Res., 20, 937-958.

Campbell, J.Y. and Viceira, L.M. (2001) Strategic Asset Allocation: Portfolio Choice for LongTerm Investors. New York: Oxford University Press.

DAYKIN, C.D. and HeY, G.B. (1990) Managing Uncertainty in a General Insurance Company. Journal of the Insitute of Actuaries, 117(2), 173-277.

Daykin, C.D., Pentikäinen, T. and Pesonen, M. (1994) Practical Risk Theory for Actuaries. Chapman and Hall.

Emms, P. and Haberman, S. (2005) Pricing general insurance using optimal control theory. Astin Bulletin, 35(2), 427-453.

Emms, P., Haberman, S. and Savoulli, I. (2007) Optimal strategies for pricing general insurance. Insurance: Mathematics \& Economics, 40(1), 15-34.

Fleming, W. and Rishel, R. (1975) Deterministic and Stochastic Optimal Control. New York: Springer Verlag.

Fleming, W.H. and Soner, H.M. (1993) Controlled Markov Processes and Viscosity Solutions. Springer-Verlag.

Friedman, A. (1964) Partial Differential Equations of Parabolic type. Prentice-Hall.

Gerber, H.U. and Pafumi, G. (1998) Utility Functions: From Risk Theory to Finance. North American Actuarial Journal, 2(3), 74-100.

HIPP, C. (2004) Stochastic Control with Application in Insurance. Working Paper, Universität Karlsruhe, Germany.

HipP, C. and Plum, M. (2000) Optimal Investment for Insurers. Insurance: Mathematics and Economics, 27, 215-228.

HøjgaARD, B. and TAKsar, M. (1997) Optimal Proportional Reinsurance Policies for Diffusion Models. Scand. Actuarial J., 2, 166-180.

Karatzas, I. and Shreve, S.E. (1998) Methods of Mathematical Finance. Springer.

KolmanovskiĬ, V.B. and Shă̌Khet, L.E. (1996) Control of Systems with Aftereffect. Translations of Mathematical Monographs, vol. 157. Americal Mathematical Society.

Lilien, G.L. and Kotler, P. (1983) Marketing Decision Making. Harper \& Row.

Merton, R.C. (1971) Optimum Consumption and Portfolio Rules in a Continuous Time Model. Journal of Economic Theory, 3, 373-413. 
Merton, R.C. (1990) Continuous-time Finance. Blackwell.

Murray, J.D. (2002) Mathematical Biology. Springer.

PentikäInen, T. (1986) Discussion of "Underwriting strategy in a competitive insurance environment". Insurance: Mathematics and Economics, 5, 81-83.

PRATT, J.W. (1964) Risk Aversion in the Small and in the Large. Econometrica, 32, 122-136.

SAmuelson, P.A. (1969) Lifetime Portfolio Selection by Dynamic Stochastic Programming. Review of Economics and Statistics, 51, 239-246.

Smith, G.D. (1985) Numerical Solution of Partial Differential Equations: Finite Difference Methods. Oxford University Press.

Taylor, G.C. (1986) Underwriting strategy in a competitive insurance environment. Insurance: Mathematics and Economics, 5(1), 59-77.

Paul EMms

Faculty of Actuarial Science and Insurance

Cass Business School

City University, London 